\title{
Dietary resource partitioning among sympatric New Zealand and Australian fur seals
}

\author{
Brad Page $^{1,2, *}$, Jane McKenzie ${ }^{1}$, Simon D. Goldsworthy ${ }^{1,2}$ \\ ${ }^{1}$ Sea Mammal Ecology Group, Zoology Department, La Trobe University, Bundoora Campus, Victoria 3086, Australia \\ ${ }^{2}$ Present address: South Australian Research and Development Institute (Aquatic Sciences), PO Box 120, Henley Beach, \\ South Australia 5022, Australia
}

\begin{abstract}
Adult male, female and juvenile New Zealand and Australian fur seals Arctocephalus forsteri and A. pusillus doriferus regularly return to colonies, creating the potential for intra- and inter-specific foraging competition in nearby waters. We hypothesise that the fur seals in this study utilise different prey, thereby reducing competition and facilitating coexistence. We analysed scats and regurgitates from adult male, female and juvenile New Zealand fur seals and adult male Australian fur seals and compared prey remains found in the samples. Most prey consumed by adult male and female fur seals occur over the continental shelf or shelf break, less than $200 \mathrm{~km}$ from Cape Gantheaume. Adult female fur seals utilised proportionally more low-energy prey such as large squid and medium-sized fish. The adult female diet reflected that of a generalist predator, dictated by prey abundance and their dependant pups' fasting abilities. In contrast, adult male New Zealand and Australian fur seals consumed proportionally more energy-rich prey such as large fish or birds, most likely because they could more efficiently access and/or handle such prey. Juvenile fur seals primarily consumed small fish that occur in pelagic waters, south of the shelf break, suggesting juveniles cannot efficiently utilise prey where adult fur seals forage. The age and sex groups in this study employ dramatically different strategies to maximise their survival and reproductive success and consequently the prey that they utilise reflect their different physiological constraints and metabolic requirements.
\end{abstract}

KEY WORDS: Arctocephalus · Fur seal · Diet - Sexual dimorphism - Competition · Eudyptula minor · Foraging ecology

Resale or republication not permitted without written consent of the publisher

\section{INTRODUCTION}

Competition for resources configures the diversity of species' sizes, behaviours, ranges and prey preferences. At its theoretical extreme, competition can lead to a species' exclusion from contested habitat (Kruuk et al. 1994). However, a more common response to interspecific competition is that individuals of 1 species modify the way they use resources, altering the niche they exploit (Loveridge \& Macdonald 2003). When individuals diversify their behaviour, prey resources or habitat use, competition is less intense and, on an evolutionary time scale, even closely related species can coexist (Loveridge \& Macdonald 2003).

Similarly, intra-specific differences in feeding, activity times and habitat requirements are often related to individuals' sex, age and social status (Sol et al. 2000). Segregation by age or social status can arise when superior individuals exclude subordinate juveniles from richer foraging sites, broadening the species' niche (Sol et al. 2000). In sexually dimorphic species, 2 hypotheses have been developed to explain how differences in resource exploitation by adult males and females have evolved (Stewart 1997). The ecological divergence hypothesis suggests that sexual dimorphism evolved as a consequence of males and females utilising different prey resources (Shine et al. 2002). In contrast, the intra-sexual competition hypothesis predicts that sexual dimorphism has evolved as a consequence of sexual selection and intra-sexual competition-for example, males segregate to increase their competitive ability against other males. This is the case 
for northern elephant seals Mirounga angustirostris, where adult males are up to 10 times larger than adult females (Stewart 1997). To attain such size, males feed in separate regions of the Pacific Ocean to females (Stewart 1997). Natural selection operates because large males out-compete small rivals for access to females in oestrus and large males also spend longer periods ashore during the breeding season, increasing their reproductive opportunities (Stewart 1997). Reduced competition is therefore best visualised in terms of individuals maximising their fitness.

This study describes the diet of 2 sympatric fur seal species and discusses intra- and inter-specific differences in the context of competition for resources. The species studied are New Zealand fur seals Arctocephalus forsteri and Australian fur seals A. pusillus doriferus, which haul out at the Cape Gantheaume Conservation Park on the south coast of Kangaroo Island (36 $\left.04^{\prime} \mathrm{S}, 137^{\circ} 28^{\prime} \mathrm{E}\right)$ (Fig. 1). New Zealand and Australian fur seals were intensively hunted for their pelts in the early 19th century (Ling 1999). Small populations survived on inaccessible islands and since commercial sealing ended, populations have been recovering and recolonising islands throughout their former range (Goldsworthy et al. 2003).

The New Zealand fur seal population at Cape Gantheaume has increased by approximately $16 \%$ per annum since monitoring began in 1987 and currently numbers about 9100 individuals (Goldsworthy et al. 2003). Their population in Australia is estimated to be 57000 (Goldsworthy et al. 2003). An itinerant population of male Australian fur seals also haul out at several localities on Kangaroo Island. At the Cape Gantheaume haul out, an average of 40 (SD = 18) and 16 $(\mathrm{SD}=11)$ Australian fur seals were recorded ashore each day in the summers 2000/01 and 2002/03, respectively (J. McKenzie unpubl. data) and up to 94 have been recorded ashore on a single day (Shaughnessy \& Dennis 2001). Australian fur seals do not breed at Cape Gantheaume, hence female and juvenile diets are not part of this study. Australian fur seals breed on islands in Bass Strait (Fig. 1), where the population is increasing ( 6 to $20 \%$ depending on the colony) and currently numbers about 100000 (R. Kirkwood et al. unpubl. data).

The only dietary information for New Zealand fur seals on Kangaroo Island is a summary of their main prey in summer (S. D. Goldsworthy unpubl. data, cited in Goldsworthy et al. 2003). This contrasts with extensive dietary reports from New Zealand (listed in Fea et al. 1999, Harcourt et al. 2002) and studies from southern Tasmania (Lake 1997) and Macquarie Island (Green et al. 1990). Likewise, there are 8 dietary reports for Australian fur seals around Bass Strait and Tasmania (Fig. 1) (listed in Goldsworthy et al. 2003), but none from the western end of the species' range-

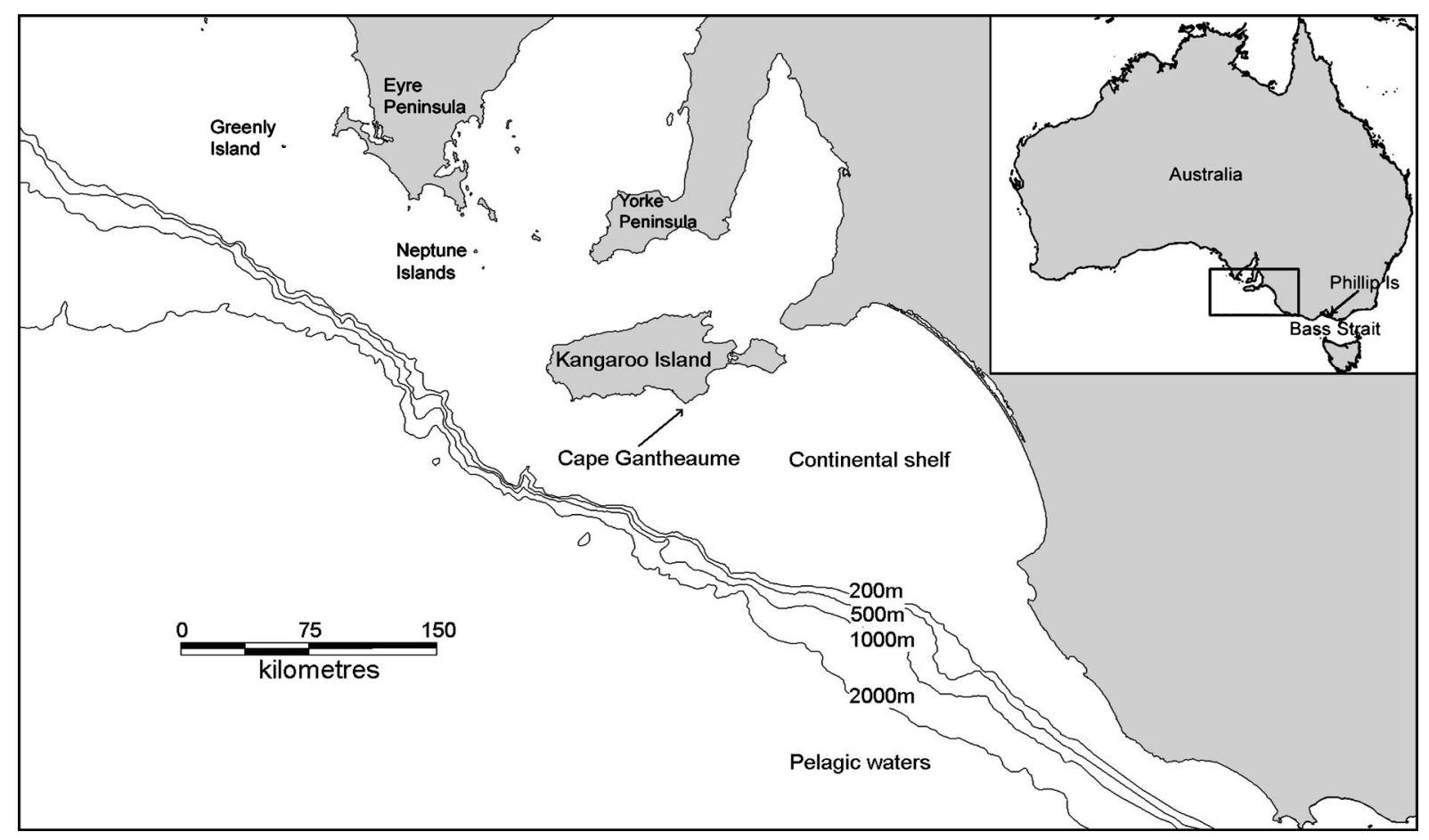

Fig. 1. Location of Cape Gantheaume and islands mentioned in the text in relation to the continental shelf, shelf break (200 to $2000 \mathrm{~m}$ depth contours) and pelagic waters 
Kangaroo Island. Previous studies demonstrate that both fur seal species target pelagic and benthic fish and cephalopods (e.g. Fea et al. 1999, Hume et al. 2004). Some New Zealand fur seal and Australian fur seal studies have identified seasonal dietary variation, with proportionally more cephalopods present in summer/autumn diet samples compared with winter/ spring samples (Gales et al. 1993, Harcourt 2001). Although spatial differences in the New Zealand fur seal's diet appear likely (reviewed in Harcourt 2001), no studies have addressed this topic by comparing samples from the same season. Regional variation in the Australian fur seal's diet was apparent between Bass Strait and southern Tasmania, with redbait Emmelichthys nitidus (southern Tasmania only), barracouta Thyrsites atun (Bass Strait only) and scorpionfish Scorpaenidae (Bass Strait only) the main taxa that contributed to the difference (Hume et al. 2004).

We aim to determine whether there is seasonal, annual, intra-specific (age and sex) and inter-specific variation in the fur seal diet. If dietary differences do exist, we aim to quantify the levels of niche overlap between age/sex/species classes of seals and discuss spatial, prey-related and life-history hypotheses for the differences. As fur seals at Cape Gantheaume segregate to an exceptional degree (authors' pers. obs., see 'Materials and methods'), we also refine dietary profiles for both species and thereby enhance seal consumption models for southern Australia.

\section{MATERIALS AND METHODS}

We analysed scats and regurgitates collected from haulouts at Cape Gantheaume, where fur seals segregate throughout the year into age, sex and species groups as follows: juvenile (15 to $25 \mathrm{~kg}$ ), adult female (30 to $55 \mathrm{~kg}$ ) and adult male (90 to $150 \mathrm{~kg}$ ) New Zealand fur seals and adult male Australian fur seals (200 to $300 \mathrm{~kg}$ ). As haulouts are located on 20 to $40 \mathrm{~m}$ wide rocky platforms, bounded by cliffs, different age/sex/species classes rarely walk though areas occupied by a particular demographic group. In addition to the location, we also used the size and diameter of scats to ensure samples were from specific demographic groups. Scats and regurgitates were collected during southern summers, autumns, winters and springs between June 2000 and February 2003. Only fresh scats and regurgitates were used, in order to minimise any bias from the samples drying out and breaking apart (Tate 1981). Each scat or regurgitate was placed in a separate plastic bag, before being frozen at $-20^{\circ} \mathrm{C}$. Prior to analysis, the samples were soaked in soapy water for at least $1 \mathrm{~d}$. Prey remains were separated by washing the samples through 1.0 and $0.5 \mathrm{~mm}$ sieves. Otoliths, eye lenses, feathers, crustacean carapaces and other bones were stored dry and cephalopod beaks were stored in $70 \%$ ethanol.

Prey remains were identified to the lowest taxonomic group by comparison with reference collections (otoliths: Dianne Furlani at CSIRO; cephalopod beaks: Museum of Victoria, Karen Evans at the University of Tasmania; feathers: Tasmanian Museum and Art Gallery; crustaceans: Centre for Fish and Fisheries Research at Murdoch University) and atlases (Smale et al. 1995, Lu \& Ickeringill 2002, S. D. Goldsworthy \& B. Page unpubl. data). Most prey were identified to species, however this was not possible for leatherjackets (grouped by Family), stargazers (Family), Gymnoscopelus spp. (Genus), Symbolophorus spp. (Genus), Beryx spp. (Genus), shearwaters (Genus), albatross (Family), jewel squid (Genus), most cuttlefish (Genus), some octopods (Genus), some redbait and jack mackerel (2 species grouped) (Table 1) and 1 unidentified fish species (2 otoliths). Taxonomic information for all prey species is provided in Table 1.

Well-preserved otoliths and cephalopod beaks were either measured using digital callipers (accurate to $0.01 \mathrm{~mm}$ ) or weighed using electronic scales (accurate to $0.0001 \mathrm{~g}$ ), depending on the regression equations available to estimate prey mass (Table 1). Upper or lower rostral length was measured for squid beaks and upper or lower hood length was measured for octopus and cuttlefish beaks. Errors associated with measurements were assessed by repeating otolith length and lower rostral length measurements 10 times, on 2 otoliths and 2 beaks.

The relative importance of each prey taxa was assessed using 3 standardised measures: (1) percentage numerical abundance (proportion of the total prey items made up by each prey taxa) and (2) percentage frequency of occurrence (proportion of samples containing a given prey taxa) (Table 2). However, as prey taxa importance in diet studies is best represented by volumetric data (Hyslop 1980), the most appropriate assessment is based on (3) percentage biomass contribution (Table 3). Prey mass was estimated using regression equations from published information and from relationships between otolith/beak measurements and fish/cephalopod mass (Table 1).

The minimum number of individuals in each sample was estimated by counting left and right otoliths, upper and lower cephalopod beaks, eye lenses, bird skulls and other bid bones and/or crustacean carapaces. Samples that contained unquantifiable remains such as teeth, leatherjacket spinal processes or feathers were recorded as containing a single individual. Samples that contained fewer identified beaks or otoliths than unidentified remains (e.g. some upper beaks, eye lenses and fish bones) were recorded as containing a 
Table 1. References for formulae and weights used to estimate the biomass contribution of each prey taxa

\begin{tabular}{|c|c|c|}
\hline Prey type & Species used for estimate & Reference for biomass estimate \\
\hline \multicolumn{3}{|l|}{ Fish } \\
\hline Anchovy (Engraulis australis) & Same species ${ }^{1}$ & Gales \& Pemberton (1990) \\
\hline Barracouta (Thyrsites atun) & Same species ${ }^{1}$ & Gales \& Pemberton (1990) \\
\hline Beryx sp. & B. splendens ${ }^{4}$ & Smale et al. (1995) \\
\hline Common bullseye (Pempheris multiradiata) & Same species $^{2}$ & D. Furlani et al. unpubl. data \\
\hline Deepwater hardyhead (Atherinason hepsetoides) & S. neopilchardus ${ }^{1}$ & Gales \& Pemberton (1990) \\
\hline Eastern school whiting (Sillago flindersi $)^{5}$ & Same species $^{2}$ & D. Furlani et al. unpubl. data \\
\hline Flathead (Neoplatycephalus sp.) & N. richardsoni ${ }^{3}$ & D. Furlani et al. unpubl. data \\
\hline Jack mackerel (Trachurus declivis) & Same species ${ }^{1}$ & $\begin{array}{l}\text { Williams \& Pullen in: } \\
\text { Gales \& Pemberton (1994) }\end{array}$ \\
\hline King George whiting (Sillaginodes puncata) ${ }^{5}$ & Same species $^{1}$ & $\begin{array}{l}\text { A. Fowler (PIRSA/SARDI) } \\
\text { unpubl. data }\end{array}$ \\
\hline Latchet (Pterygotrigla polyommata) & Same species ${ }^{1}$ & D. Furlani et al. unpubl. data \\
\hline Leatherjacket (Monocanthidae) & C. lineatus average weight & This study \\
\hline Long-finned pike (Dinolestes lewini) & Same species $^{2}$ & D. Furlani et al. unpubl. data \\
\hline Microstomatid (Nansenia macrolepis) & G. piabilis average weight & This study \\
\hline Myctophid (Gymnoscopelus sp.) & G. piabilis ${ }^{3}$ & Smale et al. (1995) \\
\hline Myctophid (Symbolophorus sp.) & S. barnardi ${ }^{1}$ & Smale et al. (1995) \\
\hline Pilchard (Sardinops sagax) & S. neopilchardus ${ }^{4}$ & Gales \& Pemberton (1990) \\
\hline Red mullet (Upeneichthys lineatus) ${ }^{5}$ & Same species average weight & Broadhurst et al. (2000) \\
\hline Red rock cod (Pseudophycis bachus) & Same species ${ }^{1}$ & Fea et al. (1999) \\
\hline Redbait (Emmelichthys nitidus) & Same species ${ }^{2}$ & $\begin{array}{l}\text { Williams \& Pullen in: } \\
\text { Gales \& Pemberton } 1994)\end{array}$ \\
\hline Redbait or jack mackerel & E. nitidus average weight ${ }^{2}$ & This study \\
\hline Silver dory (Cyttus australis) & Same species average weight & S. D. Goldsworthy unpubl. data \\
\hline Silverbelly (Parequula melbournensis) ${ }^{5}$ & Same species avg mature length & $\begin{array}{l}\text { Sarre et al. (1997), D. Furlani } \\
\text { et al. unpubl. data }\end{array}$ \\
\hline Slimy mackerel (Scomber australasicus) & Same species ${ }^{1}$ & Stewart et al. (1998) \\
\hline Southern sea garfish (Hyporhamphus melanochir) & Same species ${ }^{1}$ & Gales \& Pemberton (1990) \\
\hline Spotted warehou (Seriolella punctata) & Same species ${ }^{3}$ & D. Furlani et al. unpubl. data \\
\hline Stargazer (Uranoscopidae) & Ichthyscopus barbatus avg weight & B. Page unpubl. data \\
\hline Swallowtail (Centroberyx lineatus) & C. lineatus ${ }^{1}$ & B. Page unpubl. data \\
\hline Western Australian salmon (Arripis truttacea) & Same species ${ }^{1}$ & D. Furlani et al. unpubl. data \\
\hline Western gemfish (Rexea solandri) & Same species $^{2}$ & D. Furlani et al. unpubl. data \\
\hline Western school whiting (Sillago bassensis) ${ }^{5}$ & Same species ${ }^{4}$ & D. Furlani et al. unpubl. data \\
\hline Yellow-eye mullet (Aldrichetta forsteri) & Same species ${ }^{1}$ & D. Furlani et al. unpubl. data \\
\hline \multicolumn{3}{|l|}{ Cephalopods } \\
\hline Calamari squid (Sepioteuthis australis) lower beaks & Same species $^{3}$ & Lu \& Ickeringill (2002) \\
\hline Calamari squid ( $S$. australis) upper beaks & Same species ${ }^{1}$ & Lu \& Ickeringill (2002) \\
\hline Cuttlefish (Sepia sp.) lower beaks & Sepia sp. ${ }^{1}$ & Lu \& Ickeringill (2002) \\
\hline Giant cuttlefish (S. apama) lower beaks & Same species ${ }^{1}$ & Lu \& Ickeringill (2002) \\
\hline Gould's squid (Nototodarus gouldi) lower beaks & Same species $^{2}$ & Lu \& Ickeringill (2002) \\
\hline Maori octopus (Octopus maorum) lower beaks & Same species ${ }^{1}$ & Lu \& Ickeringill (2002) \\
\hline Octopus (Octopus sp.) lower beaks & O. berrima ${ }^{1}$ & Lu \& Ickeringill (2002) \\
\hline Octopus (Octopus sp.) upper beaks & O. berrima ${ }^{1}$ & Lu \& Ickeringill (2002) \\
\hline S. Ocean arrow squid (Todarodes filippovae) lower beaks & Same species ${ }^{2}$ & Lu \& Ickeringill (2002) \\
\hline Southern argonaut (Argonauta nodosa) upper, female & Same species ${ }^{1}$ & Lu \& Ickeringill (2002) \\
\hline Jewel squid (Histioteuthis sp.) & H. atlantica ${ }^{1}$ & Lu \& Ickeringill (2002) \\
\hline Southern keeled octopus $(O$. berrima) lower beaks & Same species ${ }^{1}$ & Lu \& Ickeringill (2002) \\
\hline \multicolumn{3}{|l|}{ Birds } \\
\hline Other birds & Sterna bergii: $350 \mathrm{~g}$ & B. Page unpubl. data \\
\hline Albatross (Diomedeidae) & Thalassarche melanophrys: $4000 \mathrm{~g}$ & B. Page unpubl. data \\
\hline Australasian gannet (Morus serrator) & Same species: $2000 \mathrm{~g}$ & A. Bunce pers. comm. \\
\hline Fleshy-footed shearwater (Puffinus carneipes) & Same species: $665 \mathrm{~g}$ & B. Page unpubl. data \\
\hline Little penguin (Eudyptula minor) & $\begin{array}{l}\text { Dec: } 1693 \mathrm{~g}, \mathrm{Jan}-\mathrm{Feb}: 914 \mathrm{~g} \text {, } \\
\text { Mar-Nov: } 1170 \mathrm{~g}\end{array}$ & S. D. Goldsworthy et al. (2000) \\
\hline Shearwater (Puffinus sp.) & P. tenuirostris: $590 \mathrm{~g}$ & B. Page unpubl. data \\
\hline \multicolumn{3}{|l|}{ Crustaceans } \\
\hline Southern velvet prawn (Metapenaeopsis palmensis) & Same species: 14.2g (max weight) & Pitcher et al. (1998) \\
\hline Burrowing shore crab (Leptograpsodes octodentatus) ${ }^{5}$ & Same species: $10 \mathrm{~g}$ & B. Page unpubl. data \\
\hline
\end{tabular}


minimum number of unidentified individuals. The unidentified biomass proportion was based on the minimum number of unidentified individuals multiplied by the average weight of individual cephalopod/fish prey consumed by the same seal-age/sex/species group in the same season.

Some studies have analysed regurgitate and scat samples separately because they can contain different proportions of cephalopod/fish remains (e.g. Hume et al. 2004). We combined data from scats and regurgitates for several reasons. Firstly, experiments on captive New Zealand fur seals indicate that representative dietary estimates can be achieved if data from scats and regurgitates are combined (Fea \& Harcourt 1997). Secondly, we collected all fresh samples from seal haulouts, in order to avoid biasing our sampling towards either scats or regurgitates. Thirdly, biomass estimates based on the minimum number of individuals per sample only require small remains (such as eye lenses or beak fragments) to be recovered to estimate prey proportions. Our study is not free of the biases inherent in scat and regurgitate analyses, however, we have attempted to minimise the effect of potential biases by utilising all identifiable prey remains to describe dietary profiles. Our assumptions are outlined and the potential biases our methods introduce are discussed below.

Semi-strong hybrid multi-dimensional scaling (MDS) was employed for multivariate analyses among age/sex/species groups, using the PATN Analysis Package (Division of Wildlife \& Ecology, CSIRO. Biomass data were not transformed because biomass data provided proportional representation and we wanted to weight dominant taxa. The Bray and Curtis association measure was used for the analysis (Beals 1984). Principal axes correlation was used to determine the most influential variables in MDS analyses. Principal axes correlation takes the variables (prey taxa) and finds the location of the best-fitted vector in the ordination space. Variables with significant correlation coefficients, r, were recorded. Stress values were calculated in 3 dimensions, to give an indication of how well the data were represented during ordination. The stress in an MDS is a measure of how distorted (or scattered) the data are after being confined to a limited number of vectors. The lower the stress value, the better the MDS representation of the data. Generally, stress values less than 0.10 are regarded as being unlikely to result in misinterpretation of the data (Schiffman et al. 1981).

To quantify dietary variation among age/sex/species groups we used the niche overlap index $(O)$ developed by Schoener (1968):

$$
O=1-0.5 \times \sum_{j=1}^{n}\left|p_{1 j}-p_{2 j}\right|
$$

where $p_{1 j}$ and $p_{2 j}$ are either the percent biomass or percent numerical abundance of the $j$ th prey taxa for each group: adult female, juvenile and adult male New Zealand fur seals, and Australian fur seals. If 2 groups consume the same prey in identical proportions, the index equals 1 . If entirely different prey are taken, the index equals 0 .

\section{RESULTS}

Of the 1197 scats and 78 regurgitates examined, $1124(94 \%)$ and $78(100 \%)$ contained prey remains, respectively. In total, 31 fish, 10 cephalopod, 5 bird and 2 crustacean taxa were identified (Table 2). A minimum of 6271 individual prey were identified in the scats and regurgitates (Table 2) with an estimated reconstructed biomass of $673 \mathrm{~kg}$ (Table 3). Biomass reconstructions were based on the estimated mass of 1440 fish, 636 cephalopods (Table 4), 148 birds and 134 crustaceans.

The average lengths and standard deviations of the 2 otoliths and 2 cephalopod beaks used to quantify measurement errors were: small otolith (redbait): $4.33 \mathrm{~mm}$ \pm 0.02 , large otolith (barracouta): $11.73 \mathrm{~mm} \pm 0.02$, small beak (Gould's squid): $3.47 \mathrm{~mm} \pm 0.09$ and large beak (Gould's squid): $9.31 \mathrm{~mm} \pm 0.11$. Converting these errors in terms of estimated reconstructed biomass, we expect our biomass estimates are: small fish weight $\pm 1.93 \%$, large fish weight $\pm 0.75 \%$, small cephalopod weight $\pm 7.60 \%$ and large cephalopod weight $\pm 3.42 \%$.

\section{Adult female New Zealand fur seal diet}

We examined 423 (89\%) scats and 53 (11\%) regurgitates from adult female New Zealand fur seals (Table 2A). Samples were analysed from 9 seasons (Tables 2A \& 3A). The number of samples from each season ranged from 32 to 90 and averaged 53 (Table $2 \mathrm{~A})$. The minimum number of individual prey in the scats and regurgitates was 2307 (Table 2A), with an estimated reconstructed biomass of $256 \mathrm{~kg}$ (Table 3A), based on the estimated mass of 471 fish, 301 cephalopods (Table 4), 35 birds and 109 crustaceans. Adult females consumed 27 fish taxa, 8 cephalopod taxa, 2 bird taxa and 1 crustacean taxa (Table 2A). To determine their most important prey, we allocated the unidentified fish and cephalopod components proportionally to the identified taxa (Table 3A). Adult females' most important prey (percent biomass greater than $4 \%$ ) were Southern Ocean arrow squid (25.8\%), Gould's squid (19.0\%), redbait (13.0\%), little penguins $(13.0 \%)$ and calamari squid $(7.9 \%)$. 


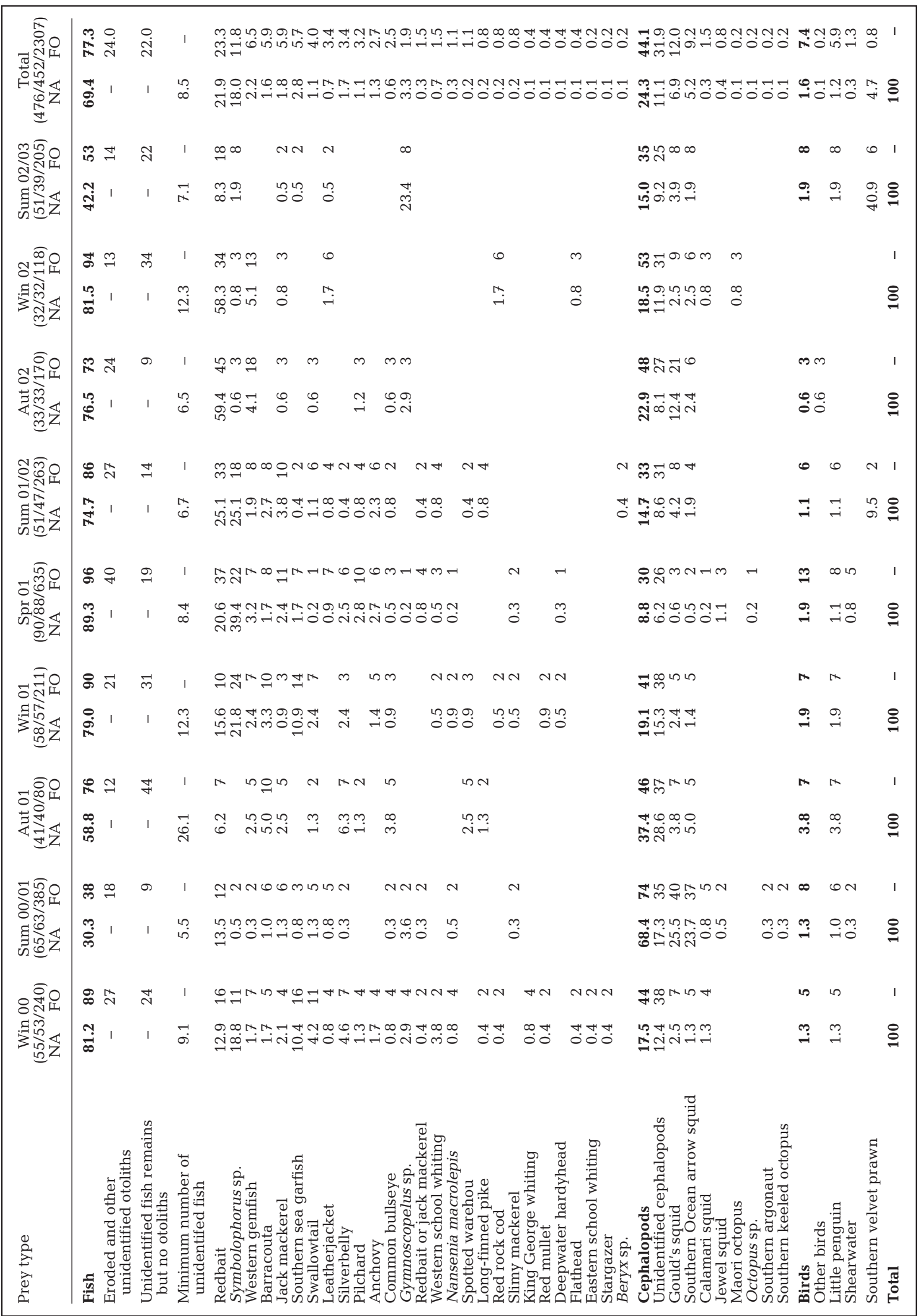




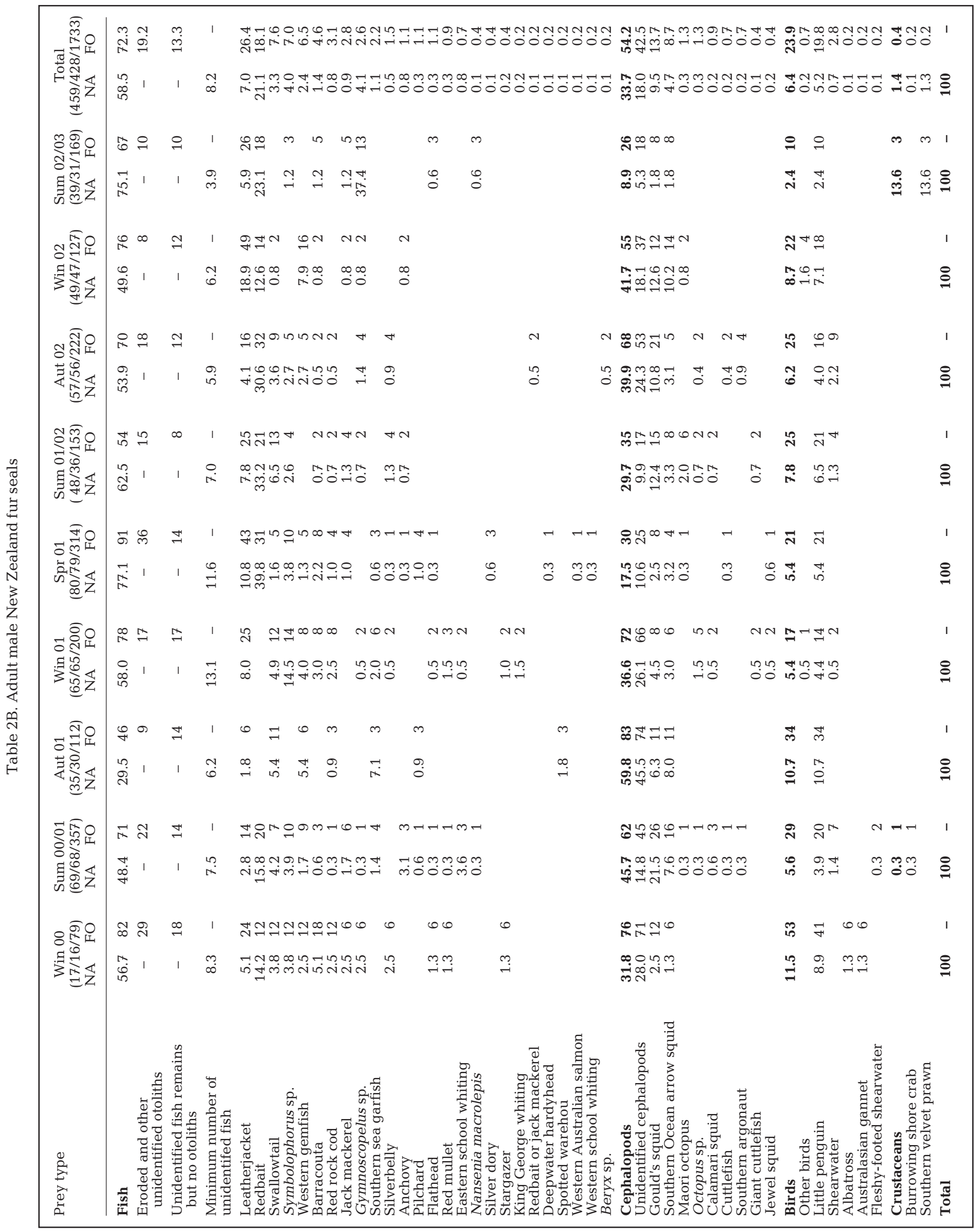

(Table 2 continued on next page) 
Table 2C. Juvenile New Zealand fur seals

\begin{tabular}{|c|c|c|c|c|c|c|c|c|c|c|c|c|c|c|}
\hline \multirow[t]{2}{*}{ Prey type } & \multicolumn{2}{|c|}{$\begin{array}{c}\text { Win } 01 \\
(23 / 21 / 336)\end{array}$} & \multicolumn{2}{|c|}{$\begin{array}{c}\text { Spr 01 } \\
(69 / 68 / 720)\end{array}$} & \multicolumn{2}{|c|}{$\begin{array}{c}\text { Sum 01/02 } \\
(45 / 41 / 247)\end{array}$} & \multicolumn{2}{|c|}{$\begin{array}{c}\text { Aut } 02 \\
(24 / 16 / 74)\end{array}$} & \multicolumn{2}{|c|}{$\begin{array}{c}\text { Win } 02 \\
(31 / 29 / 189)\end{array}$} & \multicolumn{2}{|c|}{$\begin{array}{l}\text { Sum 02/03 } \\
(20 / 20 / 106)\end{array}$} & \multicolumn{2}{|c|}{$\begin{array}{c}\text { Total } \\
(212 / 195 / 1672)\end{array}$} \\
\hline & NA & $\mathrm{FO}$ & NA & $\mathrm{FO}$ & NA & $\mathrm{FO}$ & NA & $\mathrm{FO}$ & NA & $\mathrm{FO}$ & NA & $\mathrm{FO}$ & NA & $\mathrm{FO}$ \\
\hline Fish & 97.7 & 91 & 95.5 & 96 & 75.3 & 73 & 83.7 & 67 & 89.5 & 94 & 92.5 & 95 & 91.5 & 86.8 \\
\hline Eroded and unidentified otoliths & - & 30 & - & 26 & - & 9 & - & 8 & - & 16 & - & 10 & - & 17.8 \\
\hline Unidentified fish remains but no otoliths & - & & - & 6 & - & 13 & - & & - & 6 & - & 5 & - & 6.1 \\
\hline Minimum number of unidentifed fish & 1.8 & - & 2.4 & - & 3.6 & - & 1.4 & - & 2.7 & - & 1.9 & - & 2.4 & - \\
\hline Symbolophorus sp. & 89.7 & 83 & 72.4 & 67 & 52.7 & 38 & 78.1 & 67 & 58.2 & 51 & 46.4 & 50 & 69.5 & 58.2 \\
\hline Anchovy & 1.4 & 9 & 7.5 & 20 & 6.1 & 11 & & & 1.1 & 3 & 11.3 & 25 & 5.3 & 12.7 \\
\hline Western gemfish & 1.2 & 9 & 1.9 & 12 & 2.8 & 9 & & & 2.1 & 10 & 7.5 & 20 & 2.2 & 9.9 \\
\hline Southern sea garfish & 3.3 & 13 & 1.4 & 10 & 5.7 & 7 & 1.4 & 4 & 3.2 & 10 & 0.9 & 5 & 2.6 & 8.5 \\
\hline Gymnoscopelus sp. & & & 0.6 & 3 & 4.0 & 13 & & & 16.4 & 13 & 6.6 & 20 & 3.1 & 7.5 \\
\hline Redbait & & & 3.5 & 9 & & & & & 3.7 & 13 & 17.0 & 30 & 3.0 & 7.5 \\
\hline Barracouta & & & 1.1 & 9 & & & & & 1.6 & 10 & & & 0.7 & 4.2 \\
\hline Silverbelly & 0.3 & 4 & 0.7 & 7 & & & 1.4 & 4 & & & & & 0.4 & 3.3 \\
\hline Jack mackerel & & & 0.8 & 6 & & & & & & & 0.9 & 5 & 0.4 & 2.3 \\
\hline Pilchard & & & 0.6 & 6 & & & & & & & & & 0.2 & 1.9 \\
\hline Redbait or jack mackerel & & & 0.7 & 6 & & & & & & & & & 0.3 & 1.9 \\
\hline Western school whiting & & & 1.0 & 4 & & & 1.4 & 4 & & & & & 0.5 & 1.9 \\
\hline Beryx sp. & & & 0.3 & 1 & & & & & & & & & 0.1 & 0.5 \\
\hline Common bullseye & & & 0.1 & 1 & & & & & & & & & 0.1 & 0.5 \\
\hline Eastern school whiting & & & & & & & & & 0.5 & 3 & & & 0.1 & 0.5 \\
\hline Leatherjacket & & & 0.1 & 1 & & & & & & & & & 0.1 & 0.5 \\
\hline Long-finned pike & & & 0.1 & 1 & & & & & & & & & 0.1 & 0.5 \\
\hline Deepwater hardyhead & & & 0.1 & 1 & & & & & & & & & 0.1 & 0.5 \\
\hline Western Australian salmon & & & 0.1 & 1 & & & & & & & & & 0.1 & 0.5 \\
\hline Yellow-eye mullet & & & & & 0.4 & 2 & & & & & & & 0.1 & 0.5 \\
\hline Stargazer & & & 0.1 & 1 & & & & & & & & & 0.1 & 0.5 \\
\hline Cephalopods & 2.3 & 22 & 4.1 & 20 & 24.7 & 44 & 16.3 & 17 & 10.5 & 48 & 7.5 & 20 & 8.3 & 29.0 \\
\hline Unidentified cephalopods & 2.0 & 17 & 3.1 & 16 & 14.2 & 38 & 4.1 & 8 & 7.9 & 32 & 4.7 & 15 & 5.1 & 22.1 \\
\hline Gould's squid & 0.3 & 4 & 0.6 & 3 & 6.1 & 16 & 8.1 & 13 & 2.1 & 13 & 2.8 & 10 & 2.0 & 8.9 \\
\hline Calamari squid & & & 0.1 & 1 & 0.4 & 2 & 1.4 & 4 & 0.5 & 3 & & & 0.2 & 1.9 \\
\hline Southern Ocean arrow squid & & & 0.1 & 1 & 4.0 & 4 & 2.7 & 4 & & & & & 0.8 & 1.9 \\
\hline Cuttlefish & & & 0.1 & 1 & & & & & & & & & 0.1 & 0.5 \\
\hline Jewel squid & & & 0.1 & 1 & & & & & & & & & 0.1 & 0.5 \\
\hline Little penguin & & & 0.4 & 4 & & & & & & & & & 0.2 & 1.4 \\
\hline Total & 100 & - & 100 & - & 100 & - & 100 & - & 100 & - & 100 & - & 100 & - \\
\hline
\end{tabular}

Table 2D. Adult male Australian fur seals

\begin{tabular}{|c|c|c|c|c|c|c|c|c|c|c|c|c|}
\hline \multirow[t]{2}{*}{ Prey type } & \multicolumn{2}{|c|}{$\begin{array}{c}\text { Win } 01 \\
(20 / 20 / 48)\end{array}$} & \multicolumn{2}{|c|}{$\begin{array}{c}\text { Spr 01 } \\
(40 / 39 / 80)\end{array}$} & \multicolumn{2}{|c|}{$\begin{array}{c}\text { Sum 01/02 } \\
(19 / 19 / 139)\end{array}$} & \multicolumn{2}{|c|}{$\begin{array}{c}\text { Aut } 02 \\
(24 / 24 / 167)\end{array}$} & \multicolumn{2}{|c|}{$\begin{array}{c}\text { Win } 02 \\
(25 / 25 / 125)\end{array}$} & \multicolumn{2}{|c|}{$\begin{array}{c}\text { Total } \\
(128 / 127 / 559)\end{array}$} \\
\hline & NA & $\mathrm{FO}$ & NA & $\mathrm{FO}$ & NA & $\mathrm{FO}$ & NA & $\mathrm{FO}$ & NA & $\mathrm{FO}$ & NA & $\mathrm{FO}$ \\
\hline Fish & 85.6 & 100 & 95.2 & 98 & 96.4 & 100 & 96.2 & 100 & 93.7 & 100 & 93.4 & 99.2 \\
\hline Eroded and unidentified otoliths & - & 50 & - & 48 & - & 53 & - & 25 & - & & - & 35.7 \\
\hline Unidentified fish remains but no otoliths & - & 10 & - & 10 & - & 11 & - & 29 & - & 12 & - & 14.0 \\
\hline Minimum number of unidentifed fish & 10.4 & - & 16.4 & - & 14.4 & - & 16.3 & - & 6.3 & - & 13.6 & - \\
\hline Redbait & 55.2 & 45 & 56.6 & 70 & 72.7 & 79 & 57.9 & 67 & 45.7 & 20 & 59.6 & 57.4 \\
\hline Leatherjacket & 8.8 & 55 & 9.6 & 40 & 7.9 & 58 & 7.5 & 21 & 41.7 & 80 & 11.3 & 48.8 \\
\hline Jack mackerel & 2.4 & 15 & 4.8 & 10 & & & 13.8 & 21 & & & 3.9 & 9.3 \\
\hline Swallowtail & 2.4 & 15 & & & & & 1.3 & 4 & & & 0.7 & 3.1 \\
\hline Red rock cod & 0.8 & 5 & 1.2 & 5 & 0.7 & 5 & & & & & 0.7 & 3.1 \\
\hline Symbolophorus sp. & & & 4.2 & 8 & & & & & & & 1.2 & 2.3 \\
\hline Flathead & 1.6 & 10 & 1.2 & 3 & & & & & & & 0.7 & 2.3 \\
\hline Silverbelly & 2.4 & 10 & & & & & & & & & 0.5 & 1.6 \\
\hline Stargazer & & & & & 0.7 & 5 & & & & & 0.4 & 1.6 \\
\hline Common bullseye & 0.8 & 5 & & & & & & & & & 0.2 & 0.8 \\
\hline Eastern school whiting & & & 0.6 & 3 & & & & & & & 0.2 & 0.8 \\
\hline Latchet & 0.8 & 5 & & & & & & & & & 0.2 & 0.8 \\
\hline Red mullet & & & 0.6 & 3 & & & & & & & 0.2 & 0.8 \\
\hline Cephalopods & 14.4 & 50 & 4.8 & 15 & 3.6 & 16 & 3.8 & 8 & 6.3 & 12 & 6.6 & 18.6 \\
\hline Unidentified cephalopods & 6.4 & 20 & 3.0 & 13 & 3.6 & 16 & 3.8 & 8 & 4.2 & 8 & 4.1 & 12.5 \\
\hline Octopus sp. & 0.8 & 25 & 0.6 & 3 & & & & & 2.1 & & 0.5 & 4.7 \\
\hline Gould's squid & 0.8 & 5 & & 3 & & & & & & 4 & 0.2 & 2.3 \\
\hline Cuttlefish & 0.8 & 5 & & & & & & & & & 0.2 & 0.8 \\
\hline Jewel squid & 5.6 & 5 & 1.2 & & & & & & & & 1.6 & 0.8 \\
\hline Total & 100 & - & 100 & - & 100 & - & 100 & - & 100 & - & 100 & - \\
\hline
\end{tabular}


Cephalopods comprised 29.8 to $87.9 \%$ of the reconstructed biomass per season (Table 3A). Diet summaries show Gould's squid and Southern Ocean arrow squid are more common in summer (6.8 to $64.2 \%$ biomass) and autumn (24.4 to $35.3 \%$ biomass) (Table 3A). Females consumed the 2 squid species relatively less in winter ( 0.7 to $15.0 \%$ biomass) and spring $(0.8 \%$ biomass) (Tables 2A \& 3A).

Redbait are small pelagic fish that school in shelf waters and migrate from the bottom during the day into the water column at night (Welsford \& Lyle 2003). Redbait consumption was lower in winter 2000 than in most other seasons (Table 3A), in part because the redbait consumed were small (13.6 g, SD = 12.1). Between winter 2000 and autumn 2001 redbait contributed relatively little to prey biomass (0.8 to $2.8 \%$ ) (Table $3 \mathrm{~A})$. Redbait comprised 9.5 to $31.0 \%$ of prey biomass from winter 2001 to summer 2002/03 (Table 3A). Redbait consumption was greatest in autumn (31.0\%) and winter $2002(24.1 \%)$ (Table 3A) when individual redbait averaged $46.5 \mathrm{~g}(\mathrm{SD}=35.4)$. During these 2 periods, pelagic myctophids contributed very little to prey biomass ( 0 to $0.4 \%$ ) and no penguins were taken by adult females (Tables 2A \& 3A).

Penguins contributed 4.5 to $27.3 \%$ to prey biomass each season (Table 3A). Shearwaters were consumed during summer 2000/01 (0.7\% biomass) and spring 2001 (7.4\%) (Table 3A). Prey such as calamari squid,

Table 3. Percent biomass contribution of prey taxa found in scats and regurgitates from (A) adult female, (B) adult male and (C) juvenile New Zealand fur seals and (D) adult male Australian fur seals at Cape Gantheaume. Totals for each prey type are in bold and prey taxa that averaged $<0.05 \%$ biomass are omitted

Table 3A. Adult female New Zealand fur seals

\begin{tabular}{|c|c|c|c|c|c|c|c|c|c|c|}
\hline Prey type & Win 00 & Sum $00 / 01$ & Aut 01 & Win 01 & Spr 01 & Sum $01 / 0$ & 2 Aut 02 & Win 02 & Sum $02 / 03$ & All seasons \\
\hline Fish & 29.6 & 6.9 & 19.9 & 36.4 & 42.2 & 39.8 & 36.0 & 39.9 & 17.9 & 29.8 \\
\hline Unidentified fish & 3.4 & 0.7 & 5.6 & 4.3 & 2.5 & 1.5 & 1.1 & 4.4 & 4.5 & 3.3 \\
\hline Redbait & 1.7 & 2.8 & 0.8 & 9.5 & 14.5 & 10.0 & 31.0 & 24.1 & 7.1 & 11.3 \\
\hline Barracouta & 1.5 & 0.8 & 1.7 & 5.9 & 3.0 & 9.9 & & & & 2.5 \\
\hline Jack mackerel & 1.6 & 0.6 & 2.1 & 0.9 & 6.2 & 7.4 & 0.4 & 0.4 & 1.5 & 2.3 \\
\hline Spotted warehou & & & 5.4 & 5.1 & & 2.2 & & & & 1.4 \\
\hline Swallowtail & 4.7 & 0.8 & 0.7 & 3.3 & 0.1 & 1.4 & 0.8 & & & 1.3 \\
\hline Symbolophorus sp. & 1.0 & & & 1.5 & 5.7 & 2.4 & 0.1 & 0.1 & 0.3 & 1.2 \\
\hline Flathead & 3.7 & & & & & & & 6.5 & & 1.1 \\
\hline Western gemfish & 0.5 & 0.1 & 0.6 & 1.2 & 2.4 & 0.5 & 1.6 & 1.8 & & 1.0 \\
\hline Leatherjacket & 1.2 & 0.5 & & & 2.2 & 1.1 & & 2.1 & 1.1 & 0.9 \\
\hline Pilchard & 0.7 & & 0.3 & & 1.5 & 0.4 & 0.4 & & & 0.4 \\
\hline Silverbelly & 1.2 & & 0.8 & 0.6 & 1.1 & 0.1 & & & & 0.4 \\
\hline Gymnoscopelus sp. & 0.2 & 0.1 & & & 0.1 & & 0.3 & & 3.3 & 0.4 \\
\hline Southern sea garfish & 1.2 & 0.1 & & 1.2 & 0.4 & 0.1 & & & 0.1 & 0.3 \\
\hline Common bullseye & 0.6 & 0.1 & 0.9 & 0.3 & 0.4 & 0.3 & 0.3 & & & 0.3 \\
\hline Long-finned pike & 0.7 & & 1.0 & & & 1.3 & & & & 0.3 \\
\hline Western school whiting & 1.2 & & & 0.2 & 0.2 & 0.7 & & & & 0.3 \\
\hline Slimy mackerel & & 0.2 & & 0.7 & 0.8 & & & & & 0.2 \\
\hline King George whiting & 1.7 & & & & & & & & & 0.2 \\
\hline Red mullet & 0.6 & & & 1.3 & & & & & & 0.2 \\
\hline Stargazer & 1.9 & & & & & & & & & 0.2 \\
\hline Redbait or jack mackerel & 0.1 & 0.1 & & & 0.6 & 0.2 & & & & 0.1 \\
\hline Red rock cod & & & & 0.2 & & & & 0.5 & & 0.1 \\
\hline Anchovy & 0.2 & & & 0.2 & 0.5 & 0.3 & & & & 0.1 \\
\hline Cephalopods & 56.0 & 87.9 & 60.2 & 43.9 & 29.8 & 48.3 & 61.6 & 60.1 & 45.9 & 54.9 \\
\hline Unidentified cephalopods & 33.9 & 22.6 & 35.8 & 37.1 & 27.8 & 24.2 & 26.3 & 28.1 & 39.1 & 30.6 \\
\hline Southern Ocean arrow squid & d 4.5 & 38.0 & 21.1 & 6.0 & 0.4 & 11.7 & 15.1 & 0.5 & 5.0 & 11.4 \\
\hline Gould's squid & 10.5 & 26.2 & 3.3 & 0.8 & 0.4 & 12.4 & 20.2 & 0.2 & 1.8 & 8.4 \\
\hline Calamari squid & 7.1 & 1.0 & & & 0.1 & & & 23.1 & & 3.5 \\
\hline Maori octopus & & & & & & & & 8.2 & & 0.9 \\
\hline Jewel squid & & 0.1 & & & 1.1 & & & & & 0.1 \\
\hline Birds & 14.4 & 5.2 & 19.9 & 19.7 & 28.0 & 10.5 & 2.4 & & 27.3 & 14.2 \\
\hline Other birds & & & & & & & 2.4 & & & 0.3 \\
\hline Little penguin & 14.4 & 4.5 & 19.9 & 19.7 & 20.6 & 10.5 & & & 27.3 & 13.0 \\
\hline Shearwater & & 0.7 & & & 7.4 & & & & & 0.9 \\
\hline Southern velvet prawn & & & & & & 1.4 & & & 8.9 & 1.1 \\
\hline Total (est. biomass in kg) & $100(24)$ & $100(82)$ & $100(18)$ & 100 & $100(40)$ & $100(26)$ & $100(15)$ & $100(14)$ & $100(13)$ & $100(256)$ \\
\hline
\end{tabular}


Table 3B. Adult male New Zealand fur seals

\begin{tabular}{|c|c|c|c|c|c|c|c|c|c|c|}
\hline Prey type & Win 00 & Sum 00/01 & Aut 01 & Win 01 & Spr $01 \mathrm{~S}$ & Sum 01/02 & 2 Aut 02 & Win 02 & Sum $02 / 03$ & All seasons \\
\hline Fish & 20.6 & 21.7 & 10.3 & 33.4 & 36 & 23.4 & 20.5 & 19.1 & 49.6 & 26.0 \\
\hline Unidentified fish & 1.6 & 2.1 & 1.9 & 3.2 & 3.4 & 1.8 & 2.1 & 2.3 & 2.5 & 2.1 \\
\hline Redbait & 2.1 & 5.1 & & & 12.6 & 7.9 & 9.4 & 2.6 & 25.1 & 7.2 \\
\hline Leatherjacket & 2.9 & 2.1 & 0.9 & 5.4 & 10.3 & 5.5 & 2.8 & 10.9 & 7.6 & 5.4 \\
\hline Swallowtail & 1.8 & 3.8 & 2.7 & 4 & 2 & 4.5 & 2.7 & 0.5 & & 2.4 \\
\hline Barracouta & 1.7 & 1.2 & & 7.1 & 1.4 & 0.9 & 0.6 & 0.3 & 3.1 & 1.8 \\
\hline Red rock cod & 3 & 0.6 & 0.2 & 6.5 & 2 & 1 & 0.2 & & & 1.5 \\
\hline Flathead & 3 & 1.4 & & 2 & 1.9 & & & & 4.6 & 1.4 \\
\hline Jack mackerel & 1.2 & 2 & & & 0.7 & 1.5 & & 0.4 & 3.5 & 1 \\
\hline Western gemfish & 0.4 & 1.7 & 1 & 1.1 & 0.4 & & 1.4 & 2.1 & & 0.9 \\
\hline Stargazer & 1.9 & & & 2.2 & & & 1 & & & 0.6 \\
\hline Spotted warehou & & & 3.3 & & & & & & & 0.4 \\
\hline Gymnoscopelus sp. & 0.1 & & & & & & 0.1 & & 3.1 & 0.4 \\
\hline Red mullet & 0.6 & 0.2 & & 1 & & & & & & 0.2 \\
\hline Symbolophorus sp. & 0.1 & 0.3 & & 0.5 & 0.2 & 0.1 & 0.1 & & 0.1 & 0.2 \\
\hline Eastern school whiting & & 1 & & 0.2 & & & & & & 0.1 \\
\hline Silver dory & & & & & 0.8 & & & & & 0.1 \\
\hline Silverbelly & 0.2 & & & 0.1 & 0.1 & 0.2 & 0.1 & & & 0.1 \\
\hline Southern sea garfish & & 0.1 & 0.2 & 0.1 & & & & & & 0.1 \\
\hline Pilchard & & 0.1 & 0.1 & & 0.2 & & & & & 0.1 \\
\hline Cephalopods & 23.1 & 48.2 & 47.9 & 40 & 19.9 & 41.5 & 50.6 & 45.7 & 13.2 & 36.7 \\
\hline Unidentified cephalopods & 19.9 & 20 & 34.7 & 27.6 & 15.8 & 10.7 & 26.6 & 16.6 & 10.8 & 20.4 \\
\hline Gould's squid & 1.2 & 16.6 & 5.2 & 7.2 & 0.7 & 10.3 & 18 & 7.9 & 1.8 & 7.6 \\
\hline Southern Ocean arrow squid & d 2 & 9.5 & 8 & 2.9 & 0.7 & 9.6 & 3.7 & 21 & 0.6 & 6.5 \\
\hline Giant cuttlefish & & & & 1.6 & & 7.7 & & & & 1 \\
\hline Maori octopus & & 1.3 & & & 2.4 & 1.8 & & 0.2 & & 0.6 \\
\hline Calamari squid & & 0.5 & & 0.3 & & 1.3 & & & & 0.2 \\
\hline Octopus sp. & & & & 0.2 & & 0.1 & 1.6 & & & 0.2 \\
\hline Southern argonaut & & 0.3 & & & & & 0.7 & & & 0.1 \\
\hline Jewel squid & & & & 0.2 & 0.3 & & & & & 0.1 \\
\hline Birds & 56.3 & 30.1 & 41.8 & 26.6 & 44.1 & 35.1 & 28.9 & 35.2 & 35.5 & 37.1 \\
\hline Other birds & & & & 0.8 & & & & 2.2 & & 0.3 \\
\hline Little penguin & 32.5 & 24.1 & 41.8 & 24.4 & 44.1 & 31.4 & 22.6 & 33 & 35.5 & 32.2 \\
\hline Shearwater & & 4.9 & & 1.4 & & 3.7 & 6.3 & & & 1.8 \\
\hline Albatross & 15.9 & & & & & & & & & 1.8 \\
\hline Australasian gannet & 7.9 & & & & & & & & & 0.9 \\
\hline Fleshy-footed shearwater & & 1.1 & & & & & & & & 0.1 \\
\hline Southern velvet prawn & & & & & & & & & 1.7 & 0.2 \\
\hline Total (est. biomass in kg) & $100(25)$ & $100(61)$ & $100(34)$ & 100 & $100(48)$ & $100(32)$ & $100(47)$ & 100 & $100(19)$ & $100(341)$ \\
\hline
\end{tabular}

barracouta, jack mackerel, spotted warehou, swallowtail, Symbolophorus spp. and Maori octopus contributed $>5 \%$ biomass in some seasons and averaged between 0.9 to $3.5 \%$ biomass overall (Table $3 \mathrm{~A}$ ).

\section{Adult male New Zealand fur seal diet}

Adult male New Zealand fur seal diet was determined by examining 435 (95\%) scats and 24 (5\%) regurgitates (Table 2B). Over the 9 seasons, an average of 51 samples were analysed, ranging from 17 to 80 (Table 2B). At least 1733 individual prey were identified in the scats and regurgitates (Table $2 \mathrm{~B}$ ), with an estimated reconstructed biomass of $341 \mathrm{~kg}$ (Table 3B). Biomass reconstructions were based on the estimated mass of 419 fish, 269 cephalopods (Table 4), 110 birds and 25 crustaceans. New Zealand fur seal adult males consumed 26 fish taxa, 9 cephalopod taxa, 5 bird taxa and 2 crustacean taxa (Table 2B). The reconstructed prey biomass (including unidentified prey allocation) suggested adult males' most important prey were little penguins $(32.2 \%)$, Gould's squid (17.1\%) Southern Ocean arrow squid $(14.6 \%)$, redbait $(7.9 \%)$ and leatherjacket (5.9\%).

Penguin consumption was consistent each season, making up 22.6 to $44.1 \%$ of reconstructed biomass (Table 3B). Although they were taken infrequently (Table 2B), the large mass of albatross, gannets and shearwaters resulted in some sizeable contributions to prey biomass (Table 3B). Shearwaters were consumed in summer 2000/01 (4.9\% biomass), winter 2001 
Table 3C. Juvenile New Zealand fur seals

\begin{tabular}{|c|c|c|c|c|c|c|c|}
\hline Prey type & Win 01 & Spr 01 & Sum $01 / 02$ & Aut 02 & Win 02 & Sum $02 / 03$ & All seasons \\
\hline Fish & 84.5 & 68.1 & 32.4 & 57.2 & 64.7 & 75.5 & 63.7 \\
\hline Unidentified fish & 0.1 & 0.9 & 1.3 & & 1.1 & 1.1 & 0.7 \\
\hline Symbolophorus sp. & 70.7 & 24.3 & 16 & 49.1 & 28.9 & 27 & 36 \\
\hline Western gemfish & 5.2 & 3.2 & 5 & & 6.4 & 25.8 & 7.6 \\
\hline Redbait & & 12.4 & & & 4.9 & 2.8 & 3.3 \\
\hline Anchovy & 1.7 & 3.7 & 2.7 & & 0.9 & 8.6 & 2.9 \\
\hline Southern sea garfish & 5.7 & 1.1 & 4.2 & 1.7 & 3.4 & 0.6 & 2.8 \\
\hline Gymnoscopelus sp. & & 0.3 & 1.6 & & 8.6 & 4.6 & 2.5 \\
\hline Barracouta & & 4 & & & 9 & & 2.2 \\
\hline Jack mackerel & & 2.7 & & & & 5 & 1.3 \\
\hline Stargazer & & 5.8 & & & & & 1 \\
\hline Western school whiting & & 1.5 & & 3.6 & & & 0.9 \\
\hline Silverbelly & 1.1 & 1.2 & & 2.8 & & & 0.8 \\
\hline Redbait or jack mackerel & & 2.5 & & & & & 0.4 \\
\hline Yellow-eye mullet & & & 1.6 & & & & 0.3 \\
\hline Pilchard & & 1.2 & & & & & 0.2 \\
\hline Long-finned pike & & 1 & & & & & 0.2 \\
\hline Eastern school whiting & & & & & 1.5 & & 0.2 \\
\hline Leatherjacket & & 0.9 & & & & & 0.1 \\
\hline Western Australian salmon & & 0.7 & & & & & 0.1 \\
\hline Beryx sp. & & 0.4 & & & & & 0.1 \\
\hline Common bullseye & & 0.3 & & & & & 0.1 \\
\hline Cephalopods & 15.5 & 10.4 & 67.6 & 42.8 & 35.3 & 24.5 & 32.7 \\
\hline Unidentified cephalopods & 14.8 & 8.2 & 31.9 & 18.1 & 33.3 & 20.7 & 21.2 \\
\hline Gould's squid & 0.7 & 0.9 & 14.6 & 19.4 & 1.8 & 3.8 & 6.9 \\
\hline Southern Ocean arrow squid & & 0.1 & 11.3 & 4.9 & & & 2.7 \\
\hline Calamari squid & & 1.2 & 9.8 & 0.4 & 0.2 & & 1.9 \\
\hline Little penguin & & 21.5 & & & & & 3.6 \\
\hline Total (est. biomass in kg) & $100(3)$ & $100(16)$ & $100(7)$ & $100(1)$ & $100(3)$ & $100(2)$ & $100(32)$ \\
\hline
\end{tabular}

Table 3D. Adult male Australian fur seals

\begin{tabular}{|c|c|c|c|c|c|c|}
\hline Prey type & Win 01 & Spr 01 & Sum 01/02 & Aut 02 & Win 02 & All seasons \\
\hline Fish & 93.6 & 96.9 & 96.2 & 97.3 & 97.2 & 96.2 \\
\hline Unidentified fish & 2.1 & 3.4 & 2.8 & 12.1 & 7 & 5.5 \\
\hline Redbait & 33.2 & 41 & 54 & 27.7 & 30.6 & 37.3 \\
\hline Leatherjacket & 14.1 & 17.4 & 20.2 & 28.3 & 59.6 & 27.9 \\
\hline Red rock cod & 9.2 & 15.6 & 13.2 & & & 7.6 \\
\hline Jack mackerel & 3.9 & 4.6 & & 26.1 & & 6.9 \\
\hline Flathead & 14.7 & 12.5 & & & & 5.4 \\
\hline Latchet & 9.8 & & & & & 2 \\
\hline Swallowtail & 5.4 & & & 3.1 & & 1.7 \\
\hline Stargazer & & & 6 & & & 1.2 \\
\hline Eastern school whiting & & 0.8 & & & & 0.2 \\
\hline Red mullet & & 1.1 & & & & 0.2 \\
\hline Symbolophorus sp. & & 0.5 & & & & 0.1 \\
\hline Silverbelly & 0.7 & & & & & 0.1 \\
\hline Common bullseye & 0.5 & & & & & 0.1 \\
\hline Cephalopods & 6.4 & 3.1 & 3.8 & 2.7 & 2.8 & 3.8 \\
\hline Unidentified cephalopods & 4.2 & 2.3 & 3.8 & 2.7 & 2.5 & 3.1 \\
\hline Gould's squid & 1 & 0.1 & & & 0.3 & 0.3 \\
\hline Octopus sp. & 0.4 & 0.7 & & & & 0.2 \\
\hline Cuttlefish & 0.4 & & & & & 0.1 \\
\hline Jewel squid & 0.4 & & & & & 0.1 \\
\hline Total est. biomass (kg) & $100(11)$ & $100(13)$ & $100(8)$ & $100(7)$ & $100(5)$ & $100(44)$ \\
\hline
\end{tabular}




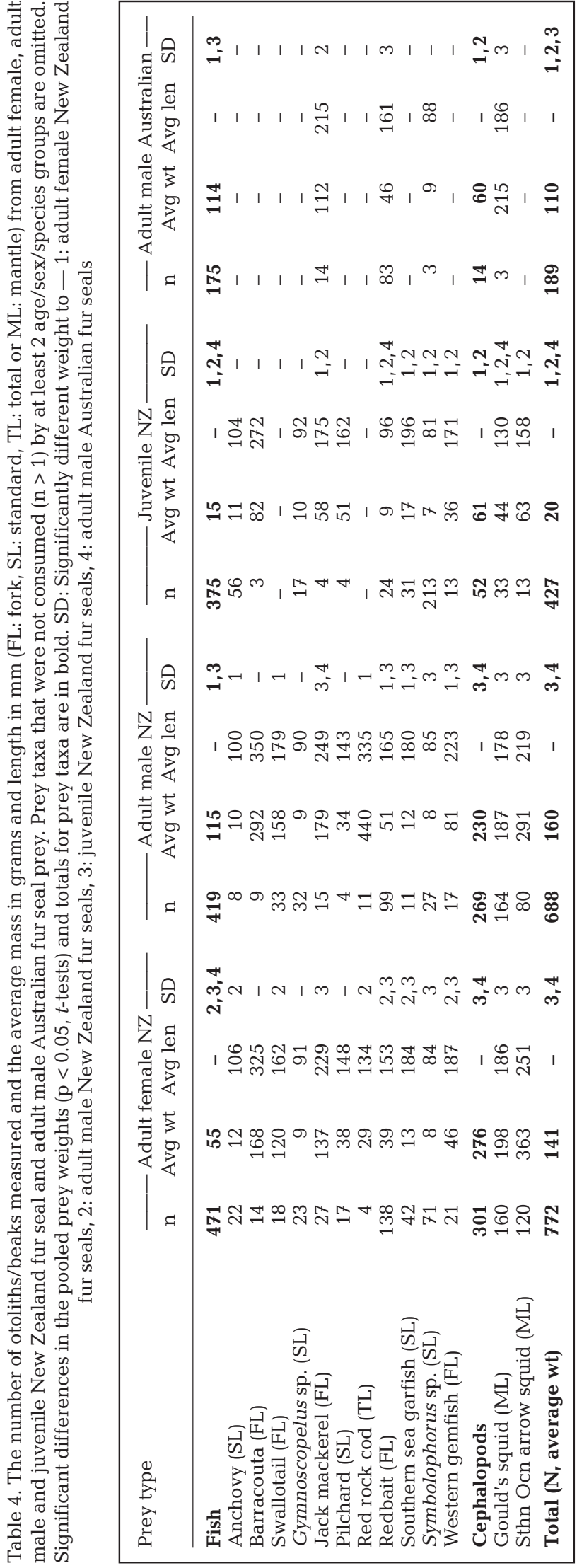

$(1.4 \%)$, summer $2001 / 02(3.7 \%)$ and autumn $(6.3 \%)$ (Table 3B).

Cephalopod biomass contribution peaked in autumn (47.9 to $50.6 \%$ biomass, Table 3B, Fig. 2) and was lowest in spring $(19.9 \%$ biomass, Table 3B, Fig. 2). Cephalopod consumption did not show clear patterns in other seasons (Table 3, Fig. 2). The autumn peaks were driven by large Gould's squid (average $=147.7 \mathrm{~g}$, $\mathrm{SD}=129$ ) and Southern Ocean arrow squid (average = $742.5 \mathrm{~g}, \mathrm{SD}=504)$, whereas individual squid were small in spring 2001 (average $=45.5 \mathrm{~g}, \mathrm{SD}=28.3$ ).

Redbait were the largest fish component in the adult males' diet (Table 3B). Redbait were identified in 15.8 to $33.2 \%$ of scats and regurgitates in the summer seasons, making up 5.1 to $25.1 \%$ of prey biomass (Table 3B). Redbait were less important prey in winter, when biomass contributions were below $2.6 \%$ (Table 3B). Redbait consumption in autumn and spring was more variable (Table 3 ). No redbait otoliths were found in adult male scats or regurgitates in autumn and winter 2001 (Table 2), suggesting they were either not present where males foraged or males targeted other prey.

Leatherjackets constituted 0.9 to $10.9 \%$ of prey biomass per season (Table 3B). Leatherjacket remains were present in all seasons with their frequency of occurrence from 6 to $49 \%$, but they were uncommon in the 2 autumn seasons (Tables 2B \& 3B). Unfortunately, only 10 species-specific remains were identified in this study, from ocean jackets Nelusetta ayraudi. Swallowtail, barracouta, red rock cod, flathead, jack mackerel and giant cuttlefish were common prey in some seasons, with respective biomass contributions averaging 1.0 to $2.4 \%$ (Table 3B).

\section{Juvenile New Zealand fur seal diet}

We analysed $211(99.5 \%)$ scats and 1 regurgitate from juvenile New Zealand fur seals (Table 2C). An average of 35 samples (range 20 to 69 ) were examined from 6 seasons (Table 2C). The minimum number of individual prey recovered was 1672 (Table 2C), with a biomass of $32 \mathrm{~kg}$ (Table 3C), based on the estimated reconstructed mass of 375 fish, 52 cephalopods (Table 4) and 3 birds. The seal's diet comprised 21 fish taxa, 5 cephalopod taxa and 1 bird taxon (Table 2). Juveniles' most important prey, based on biomass after allocation of unidentified prey, were Symbolophorus spp. (36.4\%), Gould's squid (19.6\%), Southern Ocean arrow squid $(7.7 \%)$, western gemfish $(7.7 \%)$, calamari squid $(5.4 \%)$, little penguins $(3.6 \%)$ and redbait $(3.3 \%)$.

Symbolophorus spp. and Gymnoscopelus spp. were commonly consumed by juveniles (Tables $2 \mathrm{C} \& 3 \mathrm{C}$ ). Symbolophorus spp. frequency of occurrence ranged 


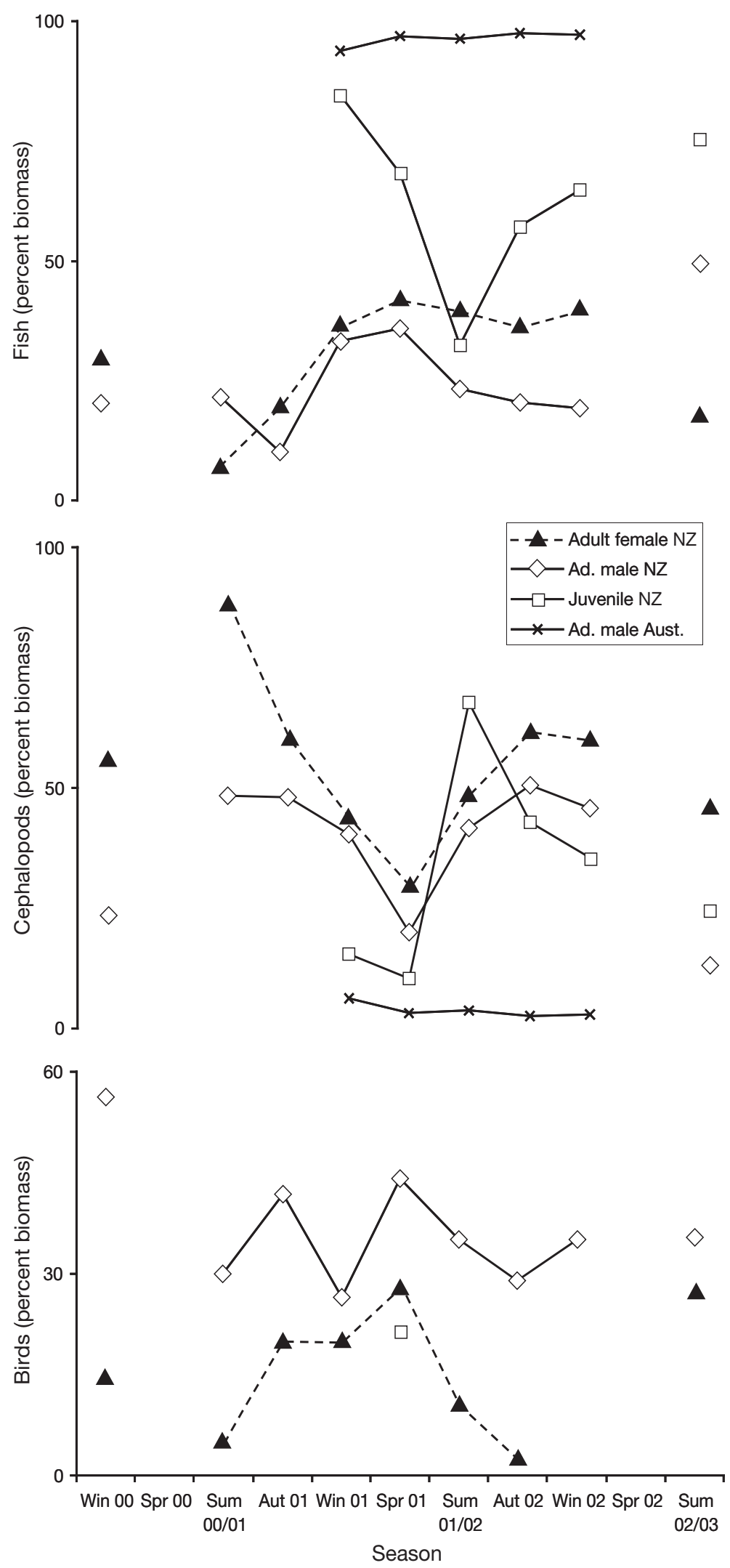

Fig. 2. Changes in the percent biomass contribution by fish, cephalopods and birds in each season for each seal age/sex/species group from 38 to $83 \%$ and they were the most numerically abundant prey, making up 46.4 to $89.7 \%$ of prey items (Table 2C). Although most common, Symbolophorus spp. contributed 16.0 to $70.7 \%$ of the biomass each season (Table 3C), owing to their small size (average $7.4 \mathrm{~g}, \mathrm{SD}=2.7$, Table 4).

Cephalopods made up 10.4 to $67.6 \%$ of the prey biomass consumed by juveniles (Table 3C). Gould's squid was the most common cephalopod prey and it was present in all seasons (Table 2C). Juvenile fur seals utilised small Gould's squid throughout the year (average $43.5 \mathrm{~g} \mathrm{SD}=43$ ) (Table 4). Southern Ocean arrow squid and calamari squid were important prey in summer 2001/02, constituting 11.3 and $9.8 \%$ of the identified prey biomass, respectively (Table 3C).

Western gemfish contributed 0 to $25.8 \%$ of prey biomass per season (Table 3C). Western gemfish were consumed in 5 of the 6 seasons and weighed an average of $35.7 \mathrm{~g}(\mathrm{SD}=13.9)$ (Tables $3 \mathrm{C} \& 4)$. Redbait were consumed in 3 of the 6 seasons and contributed 2.8 to $12.4 \%$ to prey biomass when present (Table 3C, Fig. 2). Other seasonally important juvenile prey were anchovy, southern sea garfish, Gymnoscopelus spp., barracouta and jack mackerel, with respective biomass contributions averaging 1.0 to $2.9 \%$ (Table 3C).

\section{Adult male Australian fur seal diet}

As relatively few Australian fur seals haul out at Cape Gantheaume, we collected only 128 scats and found no regurgitates (Table 2D). The number of samples analysed averaged 21 and ranged 19 to 40 over the 5 seasons (Table 2D). The minimum number of individual prey identified in the scats and regurgitates was 559 (Table 2D), with a biomass of $44 \mathrm{~kg}$ (Table 3D), based on the estimated reconstructed mass of 175 fish and 14 cephalopods (Table 4). Australian fur seals consumed 13 fish taxa and 4 cephalopod taxa (Table 2D). After allocating unidentified prey, Australian fur seals' most important prey were redbait $(39.6 \%)$, leatherjacket $(29.6 \%)$, red rock $\operatorname{cod}(8.1 \%)$, jack mackerel $(7.3 \%)$ and flathead $(5.7 \%)$.

Fish were the only important prey group for Australian fur seals at Cape Gantheaume, constituting 93.6 to $97.3 \%$ of the biomass in each season (Fig. 2). Redbait were important in all seasons, having the highest biomass contribution (37.3\% of identified prey), frequency of occurrence $(57.4 \%)$ and numerical abundance $(59.6 \%)$ (Tables 2D \& 3D). Leatherjackets were also com- 
mon prey with seasonal biomass contributions ranging 14.1 to $59.6 \%$. Red rock cod and jack mackerel were taken in winter 2001, spring 2001 and summer 2001/02, with biomass contributions ranging 9.2 to $15.6 \%$ and 3.9 to $26.1 \%$, respectively (Table 3D). Flathead were taken in winter and spring 2001, contributing 12.5 and $14.7 \%$ to prey biomass (Table 3D).

\section{Seasonal trends in fish, cephalopod and bird consumption}

Adult male New Zealand fur seals consumed a greater proportion of birds than other groups in all seasons. Adult female and male New Zealand fur seals consumed similar fish and cephalopod proportions in several seasons and some seasonal trends were apparent (Fig. 2, Table 3A,B). The fish proportion consumed by adult female and male New Zealand fur seals was similar in winter 2000 and decreased in summer 2000/01 (adult females) and autumn 2001 (adult males) (Fig. 2). The fish proportion increased in winter 2001 and spring 2001, which coincided with increased bird consumption by females and drops in cephalopod consumption by both adult female and male New Zealand fur seals (Fig. 2). Adult females and males consumed similar fish proportions from summer 2001/02 to summer 2002/03 and cephalopod consumption increased until autumn 2002 and decreased in summer 2002/03 (Fig. 2, Table 3A,B).

Adult male Australian fur seals and juvenile New Zealand fur seals did not show distinct seasonal patterns in fish, cephalopod or bird consumption (Fig. 2, Table 3C,D). Adult male Australian fur seals consumed very few cephalopods and no birds (Fig. 2). The cephalopod proportion consumed by juveniles was lowest in spring 2001, similar to trends in adult female and male New Zealand fur seals (Fig. 2). The fish proportion consumed by juveniles decreased during summer 2001/02 when cephalopod consumption rose (Fig. 2). After summer 2001/02, the fish proportion consumed by juveniles increased each season and the cephalopod proportion decreased (Fig. 2, Table 3C).

\section{Variation in the prey size taken by different age, sex and species groups}

Differences were apparent in the prey size consumed by the different age/sex/species groups (Table 4). Adult male New Zealand fur seals consumed the single largest prey from each group: albatross $(4000 \mathrm{~g})$, giant cuttlefish $(2418 \mathrm{~g})$ and barracouta (1302 g). Overall, adult male New Zealand fur seal prey was the largest (all prey taxa: average $160 \mathrm{~g}$ ) and overall they also consumed the largest fish prey $(115 \mathrm{~g})$, followed by adult male Australian fur seals (overall: $110 \mathrm{~g}$ fish: $114 \mathrm{~g}$ ) adult females (141 and $55 \mathrm{~g}$ ) and juveniles (20 and $15 \mathrm{~g}$ ) (most differences between seal age/sex/species groups were significant: Table 4). Overall, adult female and adult male New Zealand fur seals consumed the largest cephalopod prey (276 and $230 \mathrm{~g}$ ), followed by juveniles and adult male Australian fur seals (61 and $60 \mathrm{~g}$ ) (some differences are significant: Table 4).

On a taxon-by-taxon basis, adult male New Zealand fur seals generally consumed the largest fish, followed by adult females, Australian fur seals and juveniles (many differences were significant: Table 4). Australian fur seals consumed a few large cephalopods and adult female and male New Zealand fur seals consumed many large cephalopods, while juveniles took mostly smaller individuals (many differences were significant: Table 4). Juvenile New Zealand fur seals consumed significantly smaller prey than other groups in most cases (Table 4), except for southern sea garfish, which were significantly larger (Table 4 ).

\section{Age group, sex and inter-specific dietary differences}

We expected to find very little resource partitioning between seal age/sex/species groups because 39 of the 48 prey taxa were recovered from at least 2 groups. For example, redbait was common prey for all seal groups, contributing $11.3,3.3,7.2$ and $37.3 \%$ biomass for adult female, juvenile, adult male New Zealand fur seals and adult male Australian fur seals, respectively. Furthermore, of the 10 prey taxa that were unique to 1 age/ sex/species group, 7 occurred only once, and 3 occurred in 2 or 3 samples, so these taxa were not the source of substantial resource partitioning.

The multi-dimensional scaling (MDS) analysis shows dietary variation among seasons, ages, sexes and species (Fig. 3, 3D stress $=0.07$ ). The percentage biomass data used for MDS analyses (Fig. 3) were not transformed, however we also conducted MDS analyses on square-root-transformed data, which indicated similar groupings but with a slightly higher stress value (stress $=0.21$ ). As the (untransformed) stress value is less than 0.10, the MDS plot is thought to accurately represent the data (Schiffman et al. 1981). The distinct assemblages formed by adult male and juvenile New Zealand fur seals and adult male Australian fur seals indicate that their diets' showed less variation than adult females' (Fig. 3). The cluster formed by the adult female points overlapped with other groups' in many 3-dimensional orientations. Some of the prey taxa that the MDS used to discern dietary differences between seal groups were infrequently consumed (Fig. 3). How- 
ever, common prey taxa were also influential in the MDS analyses and the relatively distinct clusters formed by seal age/sex/species groups suggest that resource partitioning exists to some extent.

The niche overlap index is weighted by the number and size of prey consumed and therefore reflects differences and similarities in the relative prey mass taken by each group in each season (Fig. 4). We list the prey taxa responsible for $>10 \%$ of the reconstructed biomass differences between seal groups (averaged over all seasons). The niche overlap between adult female and adult male New Zealand fur seals was relatively high and similar in all seasons (49 to 60\%, Fig. 4), indicating that about half of their diets' comprised similar quantities of the same prey. Most variation between adult female and male New Zealand fur seals resulted from differential consumption of little penguins $(23 \%$ of the difference) and calamari squid (11\%) (Table 3A,B). The niche overlap between females and juveniles ranged from $20 \%$ in winter to $61 \%$ in spring (Fig. 4). The high overlap in spring 2001 was a result of juveniles utilising 22 different prey taxa compared with between 6 and 10 in other seasons. Large dietary differences between adult females and juveniles were due to differential predation on Symbolophorus spp. (24\%), Southern Ocean arrow squid (14\%) and little penguins (11\%) (Table 3). Niche overlap between juvenile and adult male New Zealand fur seals was lowest in winter $(22 \%)$ and similar in other seasons (37 to 50\%) (Fig. 4), resulting from differential consumption of Symbolophorus spp. (25\%), little penguins $(20 \%)$ and Southern Ocean arrow squid (14\%) (Table 3B,C).

Australian fur seals generally utilised fish taxa and fewer prey taxa overall (Table 2D) so most inter-specific niche overlap values were lower than intra-specific values (Fig. 4). The niche overlap between adult female New Zealand fur seals and adult male Australian fur seals ranged from 22 to $26 \%$ in autumn, winter and spring to $50 \%$ in summer (Fig. 4). Similarly, the niche overlap between adult male New Zealand and Australian fur seals ranged from 13 to $31 \%$ in autumn, winter and spring to $50 \%$ in summer (Fig. 4). The peaks resulted because in summer 2001/02 Australian fur seals consumed relatively few of the taxa that were infrequently consumed by adult male and female New Zealand fur seals (jack mackerel, flathead and latchet). Overall differences between adult females and adult male Australian fur seals were due to consumption of leatherjackets (16\%), Southern Ocean arrow squid (15\%) and redbait (14\%) (Table 3A,D). Differences between adult male New Zealand and Australian fur seals resulted from differential consumption of little penguins $(20 \%)$, redbait $(18 \%)$, Southern Ocean arrow squid (14\%), leatherjackets $(12 \%)$ and Gould's squid (11\%) (Table 3B,D). Juveniles and Australian fur seals foraged on very different prey, as indicated by the low niche overlap values, which ranged from $3 \%$ in autumn to $19 \%$ in spring (Fig. 4). These resulted from differential consumption of Symbolophorus spp. (20\%), redbait (20\%), leatherjackets $(15 \%)$ and Gould's squid (11\%) (Table 3C,D).

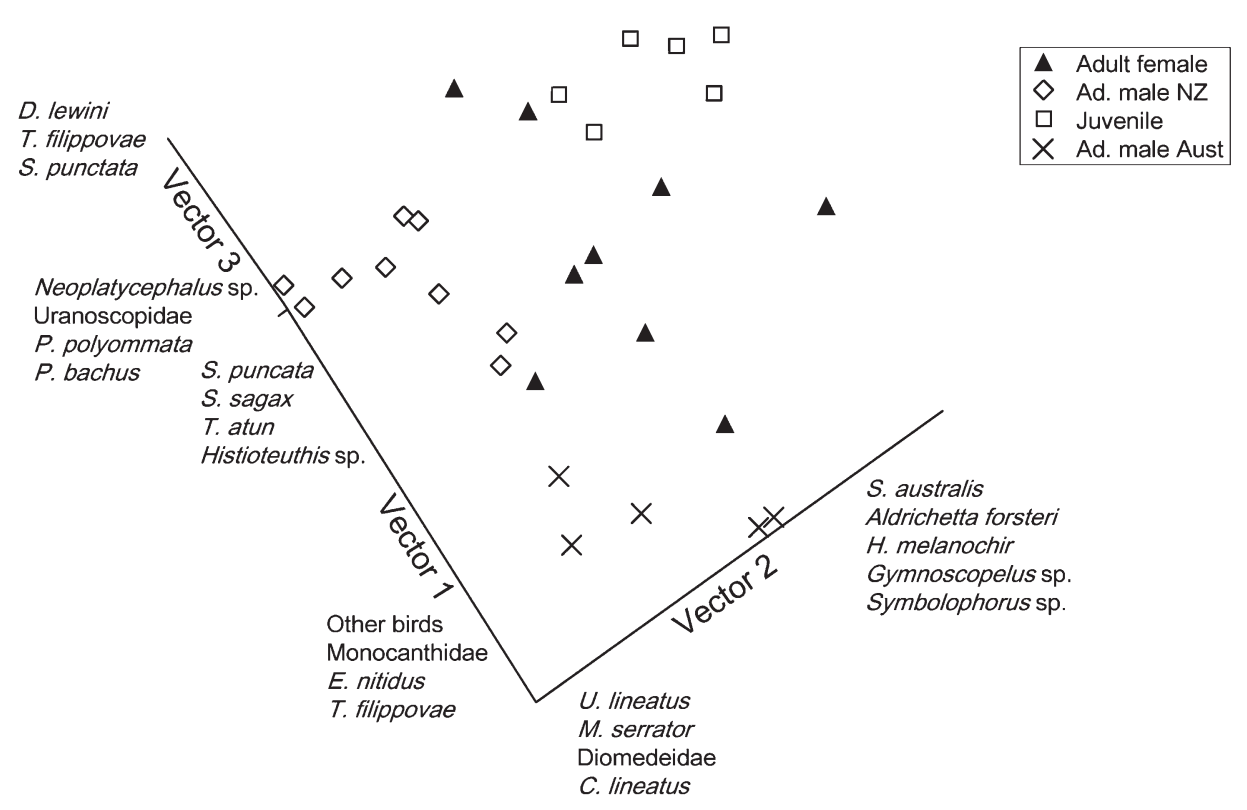

Fig. 3. Intra-specific and inter-specific variation in the diet of adult female, adult male and juvenile New Zealand fur seals and adult male Australian fur seals. Each point represents the biomass contribution in a season for an age/sex/species group. Variables (prey taxa) that had significant correlation coefficients are shown on the ends of each axis. Three-dimensional stress $=0.07$ 


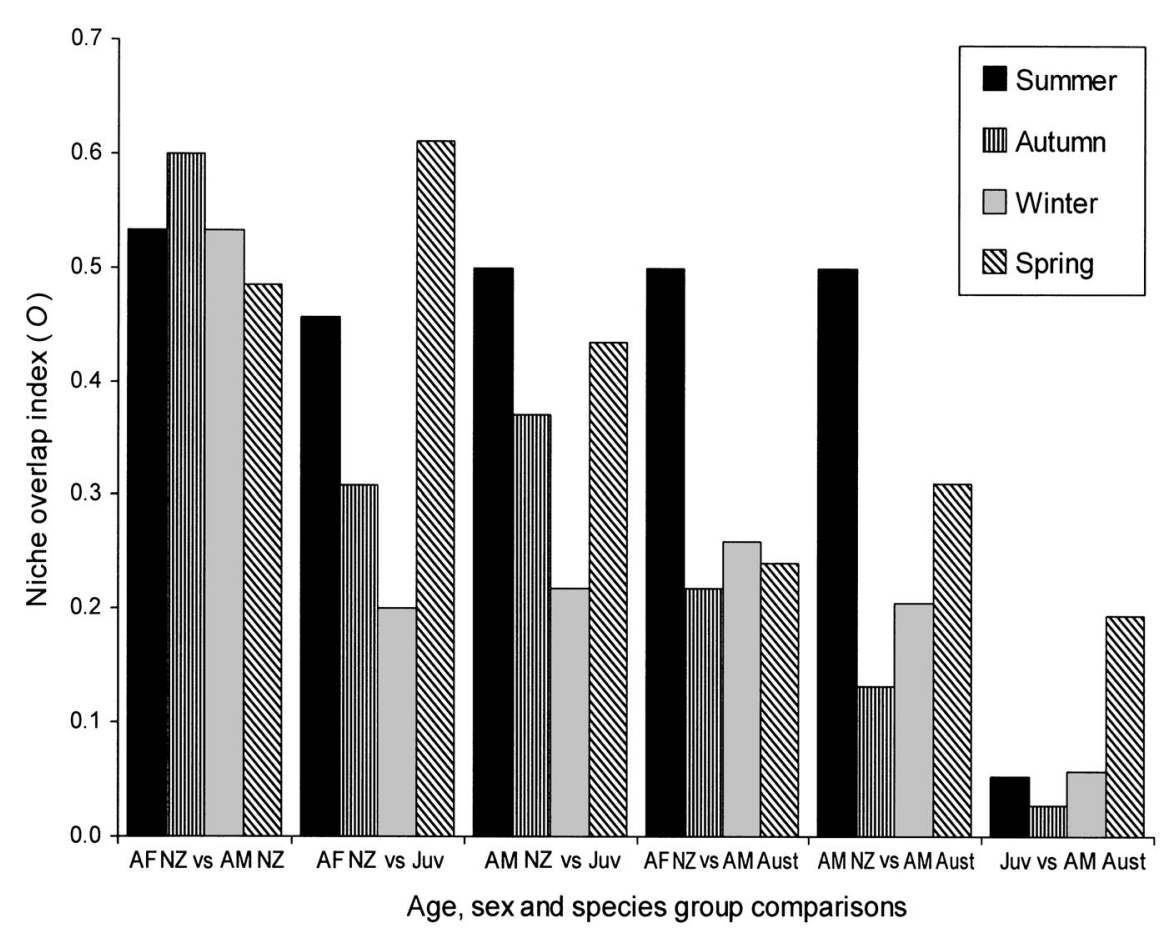

Fig. 4. Seasonal niche overlap index $(O)$ based on the percentage estimated reconstructed biomass contribution of each prey taxa consumed by each age/sex/species group. AF NZ = adult female New Zealand fur seal; $\mathrm{AM} \mathrm{NZ} \mathrm{=}$ adult male New Zealand fur seal; Juv = juvenile New Zealand fur seal: AM Aust = adult male Australian fur seal

\section{DISCUSSION}

This is the first study to quantify fur seal diet on Kangaroo Island. By targeting samples from specific demographic groups, we eliminated variation and biases caused when these groups intermingle (e.g. Hume et al. 2004).

\section{Inherent diet study biases}

Dietary validation studies conducted on captive New Zealand fur seals confirm that scat and regurgitate analyses are practical means of assessing dietary profiles (Fea \& Harcourt 1997, Lake 1997). However, all means of expressing seal diet have inherent biases due to differential digestion and voiding of prey remains, which have been discussed elsewhere for New Zealand and Australian fur seals (Gales \& Pemberton 1994, Fea \& Harcourt 1997, Lake 1997, Fea et al. 1999, Hume et al. 2004). In summary, small prey remains (such as otoliths) may be entirely digested and thereby under-represented in fur seal diet (Fea \& Harcourt 1997). Secondly, large prey remains (such as cephalopod beaks) are thought to cluster at the base of the stomach and may be retained longer than otoliths
(Gales \& Cheal 1992). As a result, we may have overestimated the consumption of large cephalopods. By combining data from scats and regurgitates and estimating the biomass of the unidentified prey we have attempted to minimise these potential errors.

In our study, quantifiable remains were rarely recovered for 2 common prey taxa - penguins and leatherjackets. Leatherjackets were identified by their bulbous spinal processes in 191 samples. Only 10 samples contained leatherjacket otoliths or dorsal spines to estimate how many had been consumed. No samples contained remains from more than 1 penguin that we could determine. As a result, penguin and leatherjacket consumption may be considerably underestimated. If this underestimate occurred for more individuals in a particular age/sex/ species class than another, it could result in misleading comparisons of biomass consumption.

Several regressions used to estimate prey biomass from otolith/beak morphometrics were based on data from other species and/or the regressions used did not encompass a small proportion of individuals that had longer or shorter predicted lengths (Table 1). Therefore, the biomass reconstructions (Fig. 2, Tables $3 \& 4$ ) should be interpreted as estimates.

Scat analysis may over-represent taxa that are consumed when seals are close to land because they may be disproportionately recovered in scats. We found 6 prey taxa that almost exclusively inhabit near-shore waters (Table 1), however, they contributed less than $2.0 \%$ of the estimated reconstructed biomass (Table 3 ). Diving behaviour data from adult male Australian fur seals and juvenile New Zealand fur seals is not available to determine whether they forage in near-shore waters. Similarly, we cannot be sure whether adult New Zealand fur seals feed while returning to land, their satellite tracks and diving behaviour suggest they do not. When commuting between foraging grounds and the colony, adult female and male New Zealand fur seals appear to cease foraging an average of 4.9 $(\mathrm{SD}=3.3)$ and $4.8(\mathrm{SD}=3.4) \mathrm{h}$ before hauling out, respectively (B. Page unpubl. data). Data from adult female Australian fur seals in Bass Strait (Fig. 1) also suggests they stop diving $3.4(\mathrm{SE}=0.3) \mathrm{h}$ before hauling out (Arnould \& Hindell 2001). From this point, dive records and satellite tracks (where available) indicate that seals swim at the surface, direct to the colony- 
behaviour consistent with rapid travel (Arnould \& Hindell 2001, B. Page unpubl. data). Because the passage half times (the time it takes for half the total recovered prey remains to reappear in captive adult New Zealand fur seals) are around 51 h (Fea \& Harcourt 1997), adult New Zealand fur seals arriving at haul-out sites would still be digesting some prey from distant foraging grounds. Data on adult male Australian fur seal diving behaviour and passage rates are not available, so prey consumed close to land may be overrepresented in this study. However, we recovered very few near-shore prey from Australian fur seals $(0.5 \%$ biomass, Tables 2D \& 3D).

\section{Competition for marine resources}

Many studies have suggested that resource partitioning prevents 1 group competitively excluding another (Loveridge \& Macdonald 2003). Sympatric giant petrels Macronectes halli and M. giganteus show substantial dietary overlap (Hunter 1983), with ecological separation possibly being maintained by temporal differences in the timing of breeding (Hunter 1987) and differences in foraging area (Gonzalez-Solis et al. 2000). Dietary separation rarely indicates that sympatric predators feed on completely different prey organisms (e.g. Gonzalez-Solis et al. 2000, Loveridge \& Macdonald 2003). Rather, predators generally utilise similar taxa in different proportions, as we demonstrated for New Zealand and Australian fur seals. We found that each age/sex/species group of seals used different sized prey and to some extent, different taxa.

Prey size and distribution also affects dietary composition among predators that differ in body size (Ashmole 1968). Not surprisingly, we demonstrated that adult seals consumed the largest prey and that juveniles consumed significantly smaller prey in most cases (Table 4). Adult seals most likely forage over shelf waters because they can efficiently handle the larger cephalopods and fishes that are most likely more abundant in these regions. Similar prey size vs. predator size patterns have been found in sympatric tern species because small terns cannot swallow deep-bodied prey, thereby reducing inter-specific competition (Hulsman 1987). In addition to diving ability constraints, juvenile seals are most likely limited by the prey size they can kill and consume underwater.

Our results indicate that juvenile New Zealand fur seals use prey in pelagic waters (particularly myctophids), whereas most adult New Zealand and Australian fur seals use prey that occur over the continental shelf and shelf break. Similar differences were demonstrated for New Zealand fur seals in Tasmania, where adult females consumed fewer pelagic myc- tophids than non-breeding seals (Lake 1997). Myctophids are small pelagic fish that perform vertical migrations toward the surface at night (Watanabe et al. 2002). The presence of myctophid fishes (Symbolophorus spp. and Gymnoscopelus spp.) in adult female samples suggests they also forage in pelagic waters, south of the shelf break (Fig. 1). Symbolophorus spp. frequency of occurrence averaged $11.8 \%$ and ranged from 0 to $24 \%$ (Table 2), suggesting that in some seasons up to $24 \%$ of females spent some time in pelagic waters (Fig. 1). Tracking results corroborate our findings: the 7 juveniles that we satellite tracked all foraged 200 to $1500 \mathrm{~km}$ south of the colony, in pelagic waters. Diving behaviour and satellite tracking data indicated that $89 \%$ of adult female New Zealand fur seals $(n=27)$ foraged over the continental shelf (max. $80 \mathrm{~m}$ deep) and $95 \%$ of adult male New Zealand fur seals $(\mathrm{n}=22)$ utilised the shelf break, down to $380 \mathrm{~m}$ (B. Page unpubl. data). In a study from Bass Strait (Fig. 1), all adult male Australian fur seal males $(n=6)$ foraged over both the continental shelf and shelf break (Kirkwood et al. 2002). Intra-specific aggression fits this pattern because the smaller juvenile seals would be the first to be forced from the richer foraging grounds exploited by adults. Similar patterns are apparent at pigeon foraging grounds where food is abundant because dominant pigeons aggressively exclude subordinates (Sol et al. 2000). However, in fur seals these spatial differences are more intuitively explained by differences in prey distribution, life-history constraints or diving ability.

A seal's body mass is correlated with its oxygen storing and diving ability, which determine how deeply prey can be accessed (Mori 2002). In pelagic waters, most cephalopods and fishes perform vertical migrations towards the surface each night, where they feed on plankton (Pearre 2003). Juvenile fur seals are unlikely to be deep divers and are probably restricted to nocturnal, visual hunting in near-surface waters. In contrast, the prey utilised by adult fur seals (with the exception of little penguins) do not generally occur near the surface and may inhabit depths that are inaccessible and/or inefficient for juvenile foraging. Juvenile phenotype, particularly their size, may constrain their ability to capture prey in these regions. The ontogeny of resource partitioning in New Zealand fur seals may be linked to the onset of puberty, when metabolic demands and growth rates increase dramatically.

The dietary differences between adult male and female fur seals are most likely shaped by their dramatically different strategies to maximise fitness. Female New Zealand fur seals nurse their pups for 10 mo before weaning. During lactation, females' foraging trips are constrained in duration by their pups' 
fasting ability, which may limit females' abilities to exploit distant foraging grounds. Female New Zealand fur seals are generalist predators, and as such, their diet is strongly influenced by both prey availability and metabolic demands of gestation and lactation (Fea et al. 1999, Harcourt et al. 2002). As predicted by central place foraging models, a lactating female's fitness is not always maximised by foraging in the most productive regions, but rather, by milk delivery rates to her dependent pup (Orians \& Pearson 1979). Females may target low-energy prey such as squid (Fig. 2) because they aggregate to spawn, allowing great quantities to be consumed in short time periods. Alternately, females may target squid because they generally comprise a higher proportion of protein than fishes (Goodman-Lowe et al. 1999), which is essential for pup growth.

Conversely, adult males' foraging trips are not constrained by dependent young, so if males made longerduration foraging trips than females they might be expected to target larger or more energy-rich prey than females. Our data indicate that male Australian fur seals target fish (Fig. 2) and male New Zealand fur seals target birds (Fig. 2), possibly because fishes and seabirds are proportionally higher in energy than cephalopods (e.g. shearwaters: Baduini et al. 2001, fishes: Goodman-Lowe et al. 1999). We interpret male fur seals' preference for energy-rich prey as support for Stewart's (1997) hypothesis that resource partitioning among male and female elephant seals results from sexual selection for large males. Fat reserves are crucial for males because they maximise their reproductive opportunities by remaining ashore during the breeding season and defending territories where females come into oestrus.

\section{Inter-specific competition between adult males}

Inter-specific dietary differences may explain why Australian fur seals attain greater sizes (average 279 kg, Warneke \& Shaughnessy 1985) than New Zealand fur seals (average 135 to $137 \mathrm{~kg}$, Troy et al. 1999). Extending our findings from New Zealand fur seals, where prey mass increased from juveniles to adults, we expected adult male Australian fur seals would consume larger prey than adult male New Zealand fur seals. In contrast, adult males of both species consumed similar sized fish prey (Table 4), perhaps limited by availability. Cephalopods consumed by Australian fur seals were significantly smaller than those consumed by New Zealand fur seals (Table 4), however cephalopods were a minor component of Australian fur seal diet (Table 3D). Similar results were found in sympatric Galápagos sea lion Zalophus californianus wollebaeki and fur seal A. galapagoensis dietary comparisons-cephalopod remains were never found in sea lion samples but they were common in fur seal samples (Dellinger \& Trillmich 1999). Adult male Australian fur seals may consume few cephalopods because they have evolved as specialised fish predators. Such prey specialisation would also explain why Australian fur seals rarely consume the penguins that they undoubtedly encounter.

Australian fur seals further specialise by using fishes that generally occur on or near the benthos, whereas adult male New Zealand fur seal prey occur throughout the water column. Diving behaviour results support these observations because most Australian fur seal dives in Bass Strait (Fig. 1) are benthic and occur throughout the day and night (Hindell \& Pemberton 1997, Arnould \& Hindell 2001). In contrast, most dives by adult male New Zealand fur seals appear to map the movements of fish and cephalopods that perform diel vertical migrations - dives only occur at night and dives are deeper at sunset and sunrise than they are around midnight (B. Page unpubl. data). Larger body size facilitates relatively longer, deeper dives (Mori 2002), enabling adult male Australian fur seals to utilise prey that are less frequently encountered by New Zealand fur seals. Conversely, this phenotypic specialisation implies that adult male Australian fur seals are less adept at foraging on mid-water prey, possibly because the development of such large body size has made them less efficient at capturing prey in the water column. Either hypothesis would explain the reduced incidence of surface and mid-water prey in the adult male Australian fur seal diet.

New Zealand fur seals recently began recolonising islands in Bass Strait and now breed sympatrically with Australian fur seals at 3 sites (Shaughnessy et al. 2002). Unfortunately, there are no diet studies from Bass Strait for the adult male Australian or the New Zealand fur seals (males or females) that facilitate dietary comparison in sympatry. Outside the breeding season, some adult male Australian fur seals forage around their colonies in Bass Strait (Kirkwood et al. 2002), however, others migrate to southern Tasmania, where they predominantly utilise benthic fishes (Hume et al. 2004). At these sites, adult male Australian fur seals are the dominant demographic group and diet studies corroborate our hypothesis of their preference for redbait and leatherjackets (Hume et al. 2004). If adult male Australian fur seals are benthic fish specialists (Hume et al. 2004, this study) and adult male New Zealand fur seals are cephalopod/penguin specialists, these preferences may have evolved in response to inter-specific competition prior to commercial sealing. 


\section{Interactions between New Zealand fur seals and little penguins}

For almost 200 yr, little penguins have existed amidst negligible predation or competition from New Zealand fur seals. Little penguin breeding distribution and population sizes may have expanded during this time. Similarly, commercial whaling in the Antarctic is thought by some researchers to have caused a 'krill surplus', which resulted in unprecedented seal and seabird populations in the region (Sladen 1964). New Zealand fur seal populations are now rapidly recovering and studies suggest that numbers may treble within the next 15 to $30 \mathrm{yr}$ (Goldsworthy et al. 2003). In other fur seals that consume birds, it is almost exclusively males that show this behaviour (Riedman 1990). Three diet studies from New Zealand also indicated that New Zealand fur seals consume birds (summarised in Harcourt 2001). On Macquarie Island, itinerant male New Zealand fur seals consume penguins (Green et al. 1990).

Although their entire breeding ranges overlap in South Australia, penguins and fur seals breed sympatrically on just 2 islands (Kangaroo \& Greenly Islands: Copley 1996, Goldsworthy et al. 2003). This may be a coincidental observation, but it is possible penguins have been made locally extinct by seals at the other localities where fur seals breed. For example, a penguin colony ( 100 pairs, S. Robinson pers. comm.) ceased to exist at Cape Gantheaume between 1992 and 2000, after New Zealand fur seals began breeding on the rocks below the penguin colony in 1992 (Shaughnessy \& Dennis 2001). Given that continued New Zealand fur seal population expansion could impact on ecologically and economically important penguin populations, there is a clear need for further research. Studies should monitor seal-penguin interactions and develop ecosystem models that simulate increased consumption of other taxa by seals to examine trophic interactions.

In studies that model ecosystem processes, ecologically and economically significant species are generally treated separately, in order to incorporate the maximum amount of information about their biology. Our data suggest that trophic models should distinguish consumption by Australian fur seals and New Zealand fur seals. Most importantly, the dietary differences among New Zealand fur seal demographic groups, which are most likely determined by life-history constraints, suggest that they should also be separated in food webs.

Acknowledgements. We thank the enthusiastic researchers who worked with us on Kangaroo Island, particularly A. Morrissey, R. McIntosh and L. Einoder. We thank A. Maguire, D. Dowie and several other National Parks staff who helped tremendously with field logistics. We thank K. Evans, J. Finn, D. Furlani, F. Hume and M. Norman for their help identifying prey remains and M. Hindell, R. Harcourt and 4 anonymous reviewers for their suggestions, which greatly improved this paper. This project was supported by Sea World Rescue and Research Foundation and the South Australian Department for Environment Wildlife Conservation Fund. B. Page received the CSIRO Marine Research scholarship and an Australian Postgraduate Award to conduct this project.

\section{LITERATURE CITED}

Arnould JPY, Hindell MA (2001) Dive behaviour, foraging locations and maternal attendance patterns of Australian fur seals (Arctocephalus pusillus doriferus). Can J Zool 79: $35-48$

Ashmole NP (1968) Body size, prey size and ecological segregation in 5 sympatric tropical terns (Aves: Laridae). Syst Zoology 17:292-304

Baduini CL, Lovvorn JR, Hunt GL (2001) Determining the body condition of short-tailed shearwaters: implications for migratory flight ranges and starvation events. Mar Ecol Prog Ser 222:265-277

Beals EW (1984) Bray Curtis ordination: an effective strategy for analysis of multivariate ecological data. Adv Ecol Res 14:1-55

Broadhurst MK, McShane PE, Larsen RB (2000) Effects of twine diameter and mesh size in the body of prawn trawls on bycatch in Gulf St. Vincent, Australia. Fish Bull 98: 463-473

Copley PB (1996) The status of seabirds in South Australia: a review. In: Ross GJB, Weaver K, Greig JC (eds) The status of Australia's seabirds. Proc Nat Seabird Workshop. Canberra. Biodiversity Group, Environment Australia

Dellinger T, Trillmich F (1999) Fish prey of the sympatric Galápagos fur seals and sea lions: seasonal variation and niche separation. Can J Zool 77:1204-1216

Fea NI, Harcourt R (1997) Assessing the use of faecal and regurgitate analysis as a means of determining fur seal diet. In: Hindell M, Kemper C (eds) Marine mammal research in the southern hemisphere, Vol 1: status, ecology and medicine. Surrey Beatty \& Sons, Sydney

Fea NI, Harcourt R, Lalas C (1999) Seasonal variation in the diet of New Zealand fur seals (Arctocephalus forsteri) at Otago Peninsula, New Zealand. Wildl Res 26:147-160

Gales NJ, Cheal AJ (1992) Estimating diet composition of the Australian sea lion Neophoca cinerea from scat analysis: an unreliable technique. Wildl Res 19:447-456

Gales R, Pemberton D (1990) Seasonal and local variation in the diet of the little penguin, Eudyptula minor, in Tasmania. Aust Wildl Res 17:231-259

Gales R, Pemberton D (1994) Diet of the Australian fur seal in Tasmania. Aust J Mar Freshw Res 45:653-664

Gales R, Pemberton D, Lu CC, Clarke MR (1993) Cephalopod diet of the Australian fur seal: variation due to location, season and sample type. Aust J Mar Freshw Res 44: $657-671$

Goldsworthy SD, Giese M, Gales RP, Brothers N, Hamill J (2000) Effects of the Iron Baron oil spill on little penguins (Eudyptula minor). II. Post-release survival of rehabilitated oiled birds. Wildl Res 27:573-582

Goldsworthy SD, Bulman C, He X, Larcombe J, Littnan C (2003) Trophic interactions between marine mammals and Australian fisheries: an ecosystem approach. In: Gales N, Hindell M, Kirkwood R (eds) Marine mammals and humans: towards a sustainable balance. CSIRO Publishing, Melbourne

Gonzalez-Solis J, Croxall JP, Wood AG (2000) Foraging parti- 
tioning between giant petrels Macronectes spp. and its relationship with breeding population changes at Bird Island, South Georgia. Mar Ecol Prog Ser 204:279-288

Goodman-Lowe GD, Carpente JR, Atkinson S, Ako H (1999) Nutrient, fatty acid, amino acid and mineral analysis of natural prey of the Hawaiian monk seal, Monachus schauinslandi. Comp Biochem Physiol A 123:137-146

Green K, Williams R, Handasyde KA, Burton HR, Shaughnessy PD (1990) Inter-specific and intra-specific differences in the diet of fur seals, Arctocephalus species (Pinnipedia: Otariidae), at Macquarie Island. Aust Mammal 13:193-200

Harcourt RG (2001) Advances in New Zealand mammalogy 1990-2000: Pinnipeds. J R Soc NZ 31:135-160

Harcourt RG, Bradshaw CJA, Dickson K, Davis LS (2002) Foraging ecology of a generalist predator, the female New Zealand fur seal. Mar Ecol Prog Ser 227:11-24

Hindell MA, Pemberton D (1997) Successful use of a translocation program to investigate diving behaviour in a male Australian fur seal, Arctocephalus pusillus doriferus. Mar Mammal Sci 13:219-228

Hulsman K (1987) Resource partitioning among sympatric species of tern. Ardea 75:255-262

Hume F, Hindell MA, Pemberton D, Gales R (2004) Spatial and temporal variation in the diet of a high trophic level predator, the Australian fur seal (Arctocephalus pusillus doriferus). Mar Biol 144:407-415

Hunter S (1983) The food and feeding of giant petrels Macronectes halli and M. giganteus at South Georgia. J Zool 200:521-538

Hunter S (1987) Species and sexual isolation mechanisms in sibling species of giant petrels Macronectes. Polar Biol 7: 295-301

Hyslop EJ (1980) Stomach contents analysis-a review of methods and their application. J Fish Biol 17:411-429

Kirkwood RJ, Gales N, Lynch M, Dann P (2002) Satellite tracker deployments on adult male Australian fur seals, Arctocephalus pusillus doriferus: methods and preliminary results. Aust Mammal 24:73-83

Kruuk H, Kanchanasaka B, O'Sullivan S, Wanghangsa S (1994) Niche separation in 3 sympatric otters Lutra perspicillata, L. lutra and Aonyx cinerea in Hai Kha Khaeng, Thailand. Biol Conserv 69:115-120

Lake S (1997) Analysis of the diet of New Zealand fur seals, Arctocephalus forsteri, in Tasmania. In: Hindell M, Kemper C (eds) Marine mammal research in the southern hemisphere, Vol 1: status, ecology and medicine. Surrey Beatty \& Sons, Sydney

Ling JK (1999) Exploitation of fur seals and sea lions from Australian, New Zealand and adjacent subantarctic islands during the eighteenth, nineteenth and twentieth centuries. Aust Zool 31:323-350

Loveridge AJ, Macdonald DW (2003) Niche separation in sympatric jackals (Canis mesomelas and Canis adustus). J Zool 259:143-153

Lu CC, Ickeringill R (2002) Cephalopod beak identification and biomass estimation techniques: tools for dietary studies of southern Australian finfishes. Museum Victoria Science Reports 5. Museum Victoria, Melbourne

Mori Y (2002) Optimal diving behaviour for foraging in relation to body size. J Evol Biol 15:269-276

Orians GH, Pearson NE (1979) On the theory of central place foraging. In: Horn DJ, Stairs BR, Mitchell RD (eds) Analysis of ecological systems. Ohio State University Press, Columbus, $\mathrm{OH}$

Pearre S (2003) Eat and run? The hunger/satiation hypothesis in vertical migration history, evidence and consequences. Biol Rev Cambridge Philos Soc 78:1-79
Pitcher TJ, Watson R, Courtney A, Pauly D (1998) Assessment of Hong Kong's inshore fishery resources. Fisheries Centre Research Reports 6(1). University of British Columbia, Vancouver

Riedman M (1990) The pinnipeds: seals, sea lions and walruses. University of California Press, Berkeley, CA

Sarre GA, Hyndes GA, Potter IC (1997) Habitat, reproductive biology and size composition of Paraquula melbournensis, a Gerreid with a temperate distribution. J Fish Biol 50: 341-357

Schiffman S, Reynolds ML, Young FW (1981) Introduction to multidimensional scaling. Academic Press, New York

Schoener T (1968) The Anolis lizards of Bimini: resource partitioning in a complex fauna. Ecology 65:1820-1827

Shaughnessy PD, Dennis T (2001) Research on New Zealand fur seals and Australian sea lions in South Australia, 2000-2001. Report to National Parks and Wildlife South Australia, Department for Environment and Heritage. CSIRO, Canberra

Shaughnessy PD, Kirkwood RJ \& Warneke RM (2002) Australian fur seals, Arctocephalus pusillus doriferus: pup numbers at lady Julia Percy Island, Victoria, and a synthesis of the species' population status. Wildl Res 29: 185-192

Shine R, Reed RN, Shetty S, Cogger HG (2002) Relationship between sexual dimorphism and niche partitioning within a clade of sea snakes (Laticaudinae). Oecologia 133:45-53

Sladen WJL (1964) The distribution of the Adélie and chinstrap penguins. In: Carrick R, Holdgate MW, Prevost J (eds) Biologie Antarctique. Hermann, Paris, pp 359-365

Smale MJ, Watson G, Hecht T (1995) Otolith atlas of Southern African marine fishes. JLB Smith Institute of Ichthyology, Grahamstown, South Africa

Sol D, Santos DM, Cuadrado M (2000) Age-related feeding site selection in urban pigeons (Columba livia): experimental evidence of the competition hypothesis. Can J Zool 78:144-149

Stewart BS (1997) Ontogeny of differential migration and sexual segregation in northern elephant seals. J Mammal 78:1101-1116

Stewart J, Ferrell DJ, Andrew NL (1998) Ageing yellowtail (Trachurus novaezelandiae) and blue mackerel (Scomber australasicus) in New South Wales: final report to the Fisheries Research and Development Corporation. NSW Fisheries Final Report Series No. 3. NSW Fisheries, Sydney

Tate ML (1981) The autumn-winter diet of the New Zealand fur seal Arctocephalus forsteri (Lesson) with special reference to its cephalopod prey. Diploma of Science Thesis, University of Otago, Dunedin

Troy SK, Mattlin R, Shaughnessy PD, Davie PS (1999) Morphology, age and survival of adult male New Zealand fur seals, Arctocephalus forsteri, in South Australia. Wildl Res 26:21-34

Warneke RM, Shaughnessy PD (1985) Arctocephalus pusillus, South African and Australian fur seal: taxonomy, evolution, biogeography and life history. In: Ling JK, Bryden MM (eds) Studies of the sea mammals in south latitudes. South Australian Museum, Adelaide

Watanabe H, Kawaguchi K, Hayashi A (2002) Feeding habits of juvenile surface-migratory myctophid fishes (family Myctophidae) in the Kuroshio region of the western North Pacific. Mar Ecol Prog Ser 236:263-272

Welsford DC, Lyle JM (2003) Redbait (Emmelichthys nitidus): a synopsis of fishery and biological data. Tech Rep Ser No. 20. Tasmanian Aquaculture \& Fisheries Institute, University of Tasmania, Hobart

Submitted: July 19, 2004; Accepted: November 4, 2004

Proofs received from author(s): May 5, 2005 